\title{
Glycolaldehyde in Perseus young solar analogs ${ }^{\star}, \star \star$
}

\author{
M. De Simone ${ }^{1}$, C. Codella ${ }^{1}$, L. Testi ${ }^{1,2,3,4}$, A. Belloche ${ }^{5}$, A. J. Maury ${ }^{6}$, S. Anderl ${ }^{7,8}$, Ph. André ${ }^{6}$, \\ S. Maret ${ }^{7,8}$, and L. Podio ${ }^{1}$ \\ 1 INAF, Osservatorio Astrofisico di Arcetri, Largo E. Fermi 5, 50125 Firenze, Italy \\ e-mail: codella@arcetri .astro.it \\ 2 ESO, Karl Schwarzchild Str. 2, 85748 Garching bei München, Germany \\ 3 Excellence Cluster "Universe", Boltzmannstr. 2, 85748 Garching bei München, Germany \\ ${ }^{4}$ Gothenburg Center for Advance Studies in Science and Technology, Department of Mathematical Sciences, \\ Chalmers University of Technology and University of Gothenburg, 41296 Gothenburg, Sweden \\ 5 Max-Planck-Institut für Radioastronomie, Auf dem Hügel 69, 53121 Bonn, Germany \\ ${ }^{6}$ Laboratoire AIM-Paris-Saclay, CEA/DSM/Irfu - CNRS - Université Paris Diderot, CE Saclay, 91191 Gif-sur-Yvette Cedex, France \\ 7 Université Grenoble Alpes, CNRS, IPAG, 38000 Grenoble, France \\ ${ }^{8}$ CNRS, IPAG, 38000 Grenoble, France
}

Received 12 November 2016 / Accepted 29 December 2016

\begin{abstract}
Context. The earliest evolutionary stages of low-mass protostars are characterised by the so-called hot-corino stage, when the newly born star heats the surrounding material and enrich the gas chemically. Studying this evolutionary phase of solar protostars may help understand the evolution of prebiotic complex molecules in the development of planetary systems.

Aims. In this paper we focus on the occurrence of glycolaldehyde $\left(\mathrm{HCOCH}_{2} \mathrm{OH}\right)$ in young solar analogs by performing the first homogeneous and unbiased study of this molecule in the Class 0 protostars of the nearby Perseus star forming region.

Methods. We obtained sub-arcsec angular resolution maps at $1.3 \mathrm{~mm}$ and $1.4 \mathrm{~mm}$ of glycolaldehyde emission lines using the IRAM Plateau de Bure (PdB) interferometer in the framework of the CALYPSO IRAM large program.

Results. Glycolaldehyde has been detected towards 3 Class 0 and 1 Class I protostars out of the 13 continuum sources targeted in Perseus: NGC 1333-IRAS2A1, NGC 1333-IRAS4A2, NGC 1333-IRAS4B1, and SVS13-A. The NGC 1333 star forming region looks particularly glycolaldehyde rich, with a rate of occurrence up to $60 \%$. The glycolaldehyde spatial distribution overlaps with the continuum one, tracing the inner 100 au around the protostar. A large number of lines (up to 18), with upper-level energies $E_{\mathrm{u}}$ from $37 \mathrm{~K}$ up to $375 \mathrm{~K}$ has been detected. We derived column densities $\geq 10^{15} \mathrm{~cm}^{-2}$ and rotational temperatures $T_{\text {rot }}$ between $115 \mathrm{~K}$ and $236 \mathrm{~K}$, imaging for the first time hot-corinos around NGC 1333-IRAS4B1 and SVS13-A.

Conclusions. In multiple systems glycolaldehyde emission is detected only in one component. The case of the SVS13-A+B and IRAS4-A1+A2 systems support that the detection of glycolaldehyde (at least in the present Perseus sample) indicates older protostars (i.e. SVS13-A and IRAS4-A2), evolved enough to develop the hot-corino region (i.e. $100 \mathrm{~K}$ in the inner 100 au). However, only two systems do not allow us to firmly conclude whether the primary factor leading to the detection of glycolaldehyde emission is the environments hosting the protostars, evolution (e.g. low value of $L_{\text {submm }} / L_{\text {int }}$ ), or accretion luminosity (high $L_{\text {int }}$ ).
\end{abstract}

Key words. stars: formation - ISM: jets and outflows - ISM: molecules

\section{Introduction}

The gas surrounding a new born star can reach temperatures higher than $100 \mathrm{~K}$, due to thermal heating by the protostar (the so called hot-corinos see e.g. Ceccarelli et al. 2007) as well as shocks produced by both accretion and ejection processes (e.g. Codella et al. 2009; Sakai et al. 2014). In turn, such temperatures favour a rich complex organic chemistry, with a consequent dramatic increase of the abundance of the so-called Complex Organic Molecules (COMs; i.e. organic molecules with at least 6 atoms detected in space, Herbst \& van Dishoeck 2009), which can be considered the first bricks to build biologically relevant molecules. Among the COMs, glycolaldehyde $\left(\mathrm{HCOCH}_{2} \mathrm{OH}\right)$ is

\footnotetext{
* Based on observations carried out with the IRAM Plateau de Bure interferometer. IRAM is supported by INSU/CNRS (France), MPG (Germany), and IGN (Spain).

$\star \star$ Reduced datacube (FITS file) is available at the CDS via anonymous ftp to cdsarc.u-strasbg. fr (130.79.128.5) or via http://cdsarc.u-strasbg.fr/viz-bin/qcat?J/A+A/599/A121
}

the simplest sugar-like molecule and it is expected to be involved in the chemical processes leading to ribose (e.g. Weber 1998; Jalbout et al. 2007), in turn the backbone of terrestrial RNA.

The first detection of interstellar glycolaldehyde was made in the hot core Sagittarius B2(N) located in the high-mass star forming region Sagittarius B2 close to the Galactic Centre (Hollis et al. 2000, 2004; Halfen et al. 2006; Requena-Torres et al. 2008). Later on, Beltrán et al. (2009) imaged glycolaldehyde emission for the first time towards a star forming region, namely the massive hot core (the scaled-up version of a hotcorino) G31.41+0.31, while Calcutt et al. (2014) reported glycolaldehyde towards further hot cores. However, the large distance of the observed high-mass star forming regions $(\geq 1 \mathrm{kpc})$ hampers (i) to assess a reliable association with one or more young stellar objects due to multiplicity; and (ii) an analysis on spatial scales comparable to that of a protoplanetary disk (i.e. $\sim 100 \mathrm{au}$ ). In addition, the study of the formation and evolution of complex organic molecules (including glycolaldehyde) in young 
Solar analogs is essential to understand the potential of formation and evolution of pre-biotic material in these systems.

Jørgensen et al. (2016) reported the first detection of glycolaldehyde emission with ALMA towards the young Solar analog low-mass Class 0 protostellar system IRAS 16293-2422. They detected 13 emission lines indicating the presence of glycolaldehyde in the warm gas (200-300 K) in the inner regions of the system, within $\sim 50$ au from each protostar. This study paved the way to the study of glycolaldehyde around Sun-like star forming regions (see also the recent work by Jørgensen et al. 2016). Indeed, Coutens et al. (2015) and Taquet et al. (2015) detected, using the IRAM PdB array, up to 8 glycolaldehyde lines towards two others well know hot-corinos associated with the NCC 1333IRAS2A and NGC 1333-IRAS4A protostars, finding temperatures higher than $100 \mathrm{~K}$. Going beyond these initial findings, it is important to perform a more systematic study to understand how common is the occurrence of glycolaldehyde around solartype protostars, and whether the presence of relatively abundant gas-phase glycolaldehyde can be linked to a specific evolutionary phase. A uniform sensitivity and high angular resolution survey of protostars is the best approach to attempt to answer these questions.

The CALYPSO $^{1}$ survey of Class 0 protostars (i.e. $10^{4}-10^{5} \mathrm{yr}$ solar analog protostars; André et al. 1993, 2000), which was carried out with the IRAM-PdB interferometer (see Maury et al. 2014 , for more details), offers a unique dataset for studying glycolaldehyde. The sources are mainly Class 0 protostars with the exception of SVS13-A which has apparently entered in the Class I stage (Lada 1987). CALYPSO includes all Solar-mass protostellar systems in the Perseus L1448 $(d=232 \pm 18 \mathrm{pc}$; Hirota et al. 2011) and NGC-1333 $(d=235 \pm 18$ pc; Hirota et al. 2008) regions. The dataset (see Sect. 2) includes high angular resolution $\left(\leq 1^{\prime \prime}\right)$ spectral maps at 1.3 and $1.4 \mathrm{~mm}$, which cover a broad range of glycolaldehyde lines and upper-level energy and are adequate for an initial statistical study. In this paper we present an analysis of these data, limited to the glycolaldehyde molecule, a detailed analysis of the continuum data will be presented in Maury et al. (in prep.), and the analysis of all complex organic molecules detected in the survey in Belloche et al. (in prep.).

The goal of the present paper is: (i) to perform a first statistical analysis of the occurrence of glycolaldehyde emission towards Class 0 protostellar sources; and (ii) to take advantage of the combination of high sensitivity and high spatial resolution provided by the CALYPSO database to detect a large number of lines covering a wide range of upper-level energy, leading to reliable estimates of the rotational (excitation) temperatures and glycolaldehyde column densities. The observations are reported in Sect. 2, while in Sect. 3 we describe the selected sample. The obtained images, the derived physical conditions, and the estimate of the glycolaldehyde abundances are reported in Sect. 4. Our conclusions are summarized in Sect. 5.

\section{Observations}

The Perseus sources (listed in Table 1) were observed at $1.3 \mathrm{~mm}$ and $1.4 \mathrm{~mm}$ with the IRAM PdB six-element array during several tracks between November 2010 and February 2012 using both the $\mathrm{A}$ and $\mathrm{C}$ configurations. The shortest and longest baselines are $19 \mathrm{~m}$ and $762 \mathrm{~m}$, respectively, allowing us to recover emission at scales from $\sim 8^{\prime \prime}$ down to $\sim 0$ '.4. The glycolaldehyde

1 http://irfu.cea.fr/Projects/Calypso lines ${ }^{2}$ were observed using the WideX backend to cover two 4-GHz spectral windows (one at $1.3 \mathrm{~mm}$ and one at $1.4 \mathrm{~mm}$ ) with a spectral resolution of $2 \mathrm{MHz}\left(\sim 2.6 \mathrm{~km} \mathrm{~s}^{-1}\right.$ at $\left.1.4 \mathrm{~mm}\right)$. The observed spectral ranges are the following: $217.0-220.5 \mathrm{GHz}$ (1.4 mm hereafter), and 229.0-233.0 GHz (1.3 mm). Calibration was carried out following standard procedures, using GILDAS$\mathrm{CLIC}^{3}$. The phase rms was $\leq 65^{\circ}$, the precipitable water vapor (PWV) was typically less than $2 \mathrm{~mm}$, the system temperatures less than $200 \mathrm{~K}$. The final uncertainty on the absolute flux scale is $\leq 15 \%$. The continuum emission was removed from the visibility tables to produce continuum-free line tables. The typical rms noise in the $2-\mathrm{MHz}$ channels was $3-9 \mathrm{mJy}^{\text {beam }}{ }^{-1}$. Images were produced using natural weighting, and restored with a clean beam of $\leq 1^{\prime \prime}$, reported, for each source and for each wavelength, in Table 1.

\section{The sample}

The sample of sources is based on all the peaks detected in the $1.3 \mathrm{~mm}$ and $1.4 \mathrm{~mm}$ continuum images of CALYPSO targets in Perseus (see Codella et al. 2014; Santangelo et al. 2015; Anderl et al. 2016; Maury et al., in prep.). We selected the sources in the Perseus complex, which is a classical laboratory to study low-mass star formation; in particular, the selected sources belong to the most active sites of current star formation in the Perseus cloud: the NGC-1333 and L1448 clusters. We observed 4 multiple systems in NGC-1333 (IRAS2A, IRAS4A, IRAS4B, and SVS13) as well as 4 protostars in L1448 (2A, NB, NA, and $\mathrm{C}$ objects), for a total number of 13 objects to be analysed to search for glycolaldehyde emission. In Table 1 we report the continuum peaks detected at $1.4 \mathrm{~mm}$ in Perseus using the CALYPSO database; for every source are reported the positions of the peaks and the systemic velocities $\left(V_{\text {sys }}\right)$ extracted from the CALYPSO dataset, the $1.4 \mathrm{~mm}$ peak flux, the internal luminosity $\left(L_{\text {int }}\right)$, and the clean beams $\left(\mathrm{HBPW}_{\text {syn }}\right)$ for each wavelength. The internal luminosities are derived using the $70 \mu \mathrm{m}$ measurements provided by the Herschel Gould Belt survey (André et al. 2010). In particular, Dunham et al. (2008), in the context of the Spitzer Space Telescope Legacy Project "From Molecular Cores to Planet Forming Disks", showed how the $70 \mu \mathrm{m}$ flux and internal luminosity of a protostar are tightly correlated. As the former is a directly observable quantity but the latter is not, this correlation gives a powerful method for estimating protostellar internal luminosities. $L_{\text {int }}$ is expected to be a more reliable probe of accretion luminosity than the bolometric luminosity $L_{\text {bol }}$ (see also Ladjelate et al., in prep.), which requires a full coverage of the Spectral Energy Distribution (SED) at high-spatial resolution $\left(8^{\prime \prime}\right)$.

The continuum map of NGC 1333-IRAS2A reveals the main protostar IRAS2-A1, plus two weaker peaks, IRAS2-A2 and IRAS2-A3 (see Codella et al. 2014). The nature of A2 and A3 is still debated, being possibly dust fragments associated with the molecular cloud and swept up by the outflows driven by the nearby IRAS2-A1 protostar (see Codella et al. 2014; Tobin et al. 2016). For the purpose of the analysis presented in this paper, we have considered these as genuine protostars, except where explicitly mentioned otherwise. The NGC-1333-IRAS4 system has two main objects: IRAS4A and IRAS4B. In turn, IRAS4A is a binary composed by two Class 0 objects, separated by

\footnotetext{
2 Spectroscopic parameters have been extracted from the Jet Propulsion Laboratory molecular database (Pickett et al. 1998), see Tables 2 to 5 .

3 http: //WWW . iram. fr/IRAMFR/GILDAS
} 
M. De Simone et al.: Glycolaldehyde in Perseus young solar analogs

Table 1. Continuum peak emission at $1.4 \mathrm{~mm}$, detected in Perseus using the CALYPSO survey, where glycolaldehyde emission has been searched for.

\begin{tabular}{|c|c|c|c|c|c|c|c|c|}
\hline \multirow[t]{2}{*}{ Source } & \multirow[t]{2}{*}{$\begin{array}{c}\alpha(\mathrm{J} 2000)^{a} \\
\left(\mathrm{~h} \mathrm{~m} \mathrm{~s}^{2}\right)\end{array}$} & \multirow[t]{2}{*}{$\begin{array}{c}\delta(\mathrm{J} 2000)^{a} \\
\quad\left({ }^{\circ},{ }^{\prime \prime}\right)\end{array}$} & \multirow[t]{2}{*}{$\begin{array}{c}V_{\text {sys }}^{a} \\
\left(\mathrm{~km} \mathrm{~s}^{-1}\right)\end{array}$} & \multirow[t]{2}{*}{$\begin{array}{l}d^{b} \\
(\mathrm{pc})\end{array}$} & \multirow[t]{2}{*}{$\begin{array}{c}F_{1.4 \mathrm{~mm}} \\
(\mathrm{~mJ} / \text { beam })\end{array}$} & \multirow[t]{2}{*}{$\begin{array}{l}L_{\text {int }}^{c} \\
\left(L_{\odot}\right)\end{array}$} & \multicolumn{2}{|c|}{$\begin{array}{l}H P B W_{\text {syn }} \\
" \times{ }^{\prime \prime}\left({ }^{\circ}\right)\end{array}$} \\
\hline & & & & & & & $1.4 \mathrm{~mm}$ & $1.3 \mathrm{~mm}$ \\
\hline L1448-2A & $03: 25: 22.406$ & +304513.28 & +4.2 & 232 & 31 & 2.7 & $0^{\prime \prime} .99 \times 0^{\prime \prime} .71\left(28^{\circ}\right)$ & $11^{\prime \prime} 03 \times 0^{\prime \prime} .81\left(39^{\circ}\right)$ \\
\hline L1448-NB & $03: 25: 36.364$ & +304514.84 & +4.7 & 232 & 25 & 4.1 & $0^{\prime \prime} 81 \times 0^{\prime \prime} 67\left(67^{\circ}\right)$ & $0^{\prime \prime} 65 \times 0^{\prime \prime} .48\left(48^{\circ}\right)$ \\
\hline L1448-NA & 032536.503 & +304521.87 & +4.7 & 232 & 139 & - & $0^{\prime \prime} .81 \times 00^{\prime \prime} 67\left(67^{\circ}\right)$ & $0^{\prime \prime} 65 \times 0^{\prime \prime} .48\left(48^{\circ}\right)$ \\
\hline L1448-C & 032538.878 & +304405.32 & +5.2 & 232 & 102 & 6.8 & $0^{\prime \prime} .77 \times 0^{\prime \prime} 63\left(22^{\circ}\right)$ & $0^{\prime \prime} 61 \times 0^{\prime \prime} .38\left(33^{\circ}\right)$ \\
\hline IRAS2A3 & 032855.514 & +311434.84 & +7.3 & 235 & 16 & - & $0^{\prime \prime} 82 \times 0^{\prime \prime} 80\left(32^{\circ}\right)$ & $0^{\prime \prime} .75 \times 0^{\prime \prime} .74\left(168^{\circ}\right)$ \\
\hline IRAS2A1 & 032855.575 & +31 1437.05 & +7.3 & 235 & 99 & 26.7 & $0^{\prime \prime} 82 \times 0^{\prime \prime} .80\left(32^{\circ}\right)$ & $0^{\prime \prime} .75 \times 0^{\prime \prime} .74\left(168^{\circ}\right)$ \\
\hline IRAS2A2 & 032855.677 & +311435.56 & +7.3 & 235 & 12 & - & $0^{\prime \prime} .82 \times 0^{\prime \prime} .80\left(32^{\circ}\right)$ & $0^{\prime \prime} .75 \times 0^{\prime \prime} .74\left(168^{\circ}\right)$ \\
\hline SVS13-B & 032903.075 & +311551.71 & +8.4 & 235 & 122 & $1-2^{c}$ & $1^{\prime \prime} .06 \times 00^{\prime \prime} 80\left(20^{\circ}\right)$ & $0^{\prime \prime} .70 \times 0^{\prime \prime} .42\left(20^{\circ}\right)$ \\
\hline SVS13-A & 032903.759 & +311603.74 & +8.4 & 235 & 123 & 24.5 & $1^{\prime \prime} .06 \times 00^{\prime \prime} 80\left(20^{\circ}\right)$ & $0^{\prime \prime} .70 \times 0^{\prime \prime} .42\left(20^{\circ}\right)$ \\
\hline IRAS4A2 & 032910.429 & +311332.10 & +7.2 & 235 & 284 & - & $1^{\prime \prime} .10 \times 0^{\prime \prime} .81\left(20^{\circ}\right)$ & $0^{\prime \prime} .70 \times 0^{\prime \prime} .42\left(20^{\circ}\right)$ \\
\hline IRAS4A1 & 032910.531 & +31 1330.96 & +7.2 & 235 & 795 & 2.8 & $1^{\prime \prime} .10 \times 00^{\prime \prime} 81\left(20^{\circ}\right)$ & $0^{\prime \prime} .70 \times 0^{\prime \prime} .42\left(20^{\circ}\right)$ \\
\hline IRAS4B1 & 032912.012 & +311308.07 & +6.7 & 235 & 449 & 1.0 & $1^{\prime \prime} .08 \times 00^{\prime \prime} 83\left(20^{\circ}\right)$ & $0^{\prime \prime} .70 \times 0^{\prime \prime} .42\left(20^{\circ}\right)$ \\
\hline IRAS4B2 & 032912.840 & +311306.98 & +6.7 & 235 & 53 & - & $1^{\prime \prime} .08 \times 00^{\prime \prime} 83\left(20^{\circ}\right)$ & $0^{\prime \prime} .70 \times 0^{\prime \prime} .42\left(20^{\circ}\right)$ \\
\hline
\end{tabular}

Notes. ${ }^{(a)}$ Positions of the $1.3 \mathrm{~mm}$ continuum peak emission and systemic velocities are extracted from the CALYPSO dataset (Codella et al. 2014; Santangelo et al. 2015; Anderl et al. 2016; Maury et al., in prep.). ${ }^{(b)}$ From Hirota et al. $(2008,2011) .{ }^{(c)}$ Internal luminosities were derived from the observed flux at $70 \mu \mathrm{m}$ (Dunham et al. 2008), a wavelength at which Herschel Gould Belt survey observations (André et al. 2010) provide data at 8 " spatial resolution (see also Ladjelate et al., in prep.). The internal luminosity of SVS13-B is more uncertain due to the proximity to SVS13-A.

1".8: IRAS4-A1 and IRAS4-A2 (e.g. Looney et al. 2000). While IRAS4-A1 is more than three times brighter in the mm-flux than its companion, only IRAS4-A2 shows a high molecular complexity (see Taquet et al. 2015; Coutens et al. 2015; Santangelo et al. 2015). IRAS4B is located $\sim 30^{\prime \prime}$ southeast of IRAS4A, and it is associated with two compact continuum sources, B1 and B2, with an angular separation of $11^{\prime \prime}$ (e.g. Looney et al. 2000; Choi et al. 2011). NGC 1333-SVS13 is a cluster dominated at millimeter wavelengths by two sources: SVS13-A, and SVS13-B. SVS13A is a protostar already in the Class I stage and drives an extended outflow associated with the well-known HH7-11 chain (e.g. Lefloch et al. 1998; Chen et al. 2009). On the other hand, SVS13-B is an earlier protostar lying southwest of SVS13-A at a distance of $15^{\prime \prime}$ (e.g. Bachiller et al. 1998; Looney et al. 2000). Finally, the L1448-NA, L1448-NB, and L1448-2A protostars are located in the northern portion of the L1448 complex, at about $\sim 1^{\circ}$ southwest of NGC 1333, while L1448-C is located at the center of the L1148 complex (see Looney et al. 2000; Tobin et al. 2016, and references therein).

\section{Results and discussion}

The results of the present search towards the coordinates listed in Table 1 indicates the presence of glycolaldehyde towards 4 out of the 13 observed continuum peaks. If we consider that the classification of the IRAS2-A3 and IRAS2-A2 continuum peaks as genuine protostars is debated (see the recent paper by Tobin et al. 2016), the detection rate is $\sim 36 \%$. Interestingly, glycolaldehyde has only been detected towards the NGC 1333 sources, where the detection rate is as high as $\sim 60 \%$.

Figures A.1 to A.9 report the spectra at the frequencies of the glycolaldehyde lines observed towards IRAS2A1, SVS13A, IRAS4A2, and IRAS4B1. The spectral resolution does not allow us to probe the profiles of the emission lines and the observed spectra are affected by line blending. Nevertheless, there is little doubt that the large WideX bandwidths $(8 \mathrm{GHz}$ in total) allowed us to detect towards the four sources a considerable number of glycolaldehyde lines (up to 18) with a signal-to-noise
Table 2. Number of glycolaldehyde lines and results of the LTE analysis for the detected sources.

\begin{tabular}{lcccc}
\hline \hline Source & $N_{\text {trans }}$ & $\begin{array}{c}E_{\mathrm{u}} \\
(\mathrm{K})\end{array}$ & $\begin{array}{c}T_{\text {rot }} \\
(\mathrm{K})\end{array}$ & $\begin{array}{c}N_{\text {tot }} \\
\left(10^{14} \mathrm{~cm}^{-2}\right)\end{array}$ \\
\hline IRAS2A1 & 13 & $37-318$ & $159(24)$ & $46(20)$ \\
IRAS4A2 & 18 & $37-375$ & $236(74)$ & $88(70)$ \\
IRAS4B1 & 13 & $37-271$ & $152(35)$ & $15(9)$ \\
SVS13-A & 11 & $37-375$ & $115(13)$ & $21(8)$ \\
\hline
\end{tabular}

Notes. In several cases ( 3 for IRAS2A1, 1 for IRAS4A2, IRAS4B1, and SVS13-A; see Tables A.1 to A.4), the detected lines are due to two glycolaldehyde transitions at frequencies blended at the present spectral resolution.

ratio $(\mathrm{S} / \mathrm{N})$ of at least 3 . We then selected only those transitions with a corresponding synthetic line (derived using the rotation diagram solutions, see below) that reproduces at least $50 \%$ of a detected peak temperature. The lines were identified using the Jet Propulsion Laboratory (JPL) molecular database (Marstokk \& Møllendal 1970a, 1973; Pickett et al. 1998; Butler et al. 2001; Widicus Weaver et al. 2005; Carroll et al. 2010). Figure 1 shows examples of glycolaldehyde emission lines (in $T_{\mathrm{B}}$ scale) observed in the $1.3 \mathrm{~mm}$ and $1.4 \mathrm{~mm}$ spectral windows towards the four detected sources. Figures A.1, A.3, A.5, and A.7 show all the lines used for the following analysis. Tables A.1-A.4 report the observed line parameters; we list both peak line intensities $\left(T_{\text {peak }}\right)$ and velocities ( $V_{\text {peak }}$, consistent with the LSR velocities of the objects), linewidth (FWHM), and the integrated emission $\left(\int T \mathrm{~d} v\right)$. All the values are in $T_{\mathrm{B}}$ scale and are derived from Gaussian fits. A wide upper-level energy range is observed (see Table 2), with $E_{\mathrm{u}}$ from $37 \mathrm{~K}$ up to $375 \mathrm{~K}$ : this allows us (i) to improve on the results of Coutens et al. (2015) and Taquet et al. (2015) on IRAS2A1 and IRAS4A2, by increasing the number of detected lines and expanding the range of upper-level energy probed; and (ii) to firmly assess, for the first time, the glycolaldehyde occurrence towards IRAS4B1 and SVS13-A. 

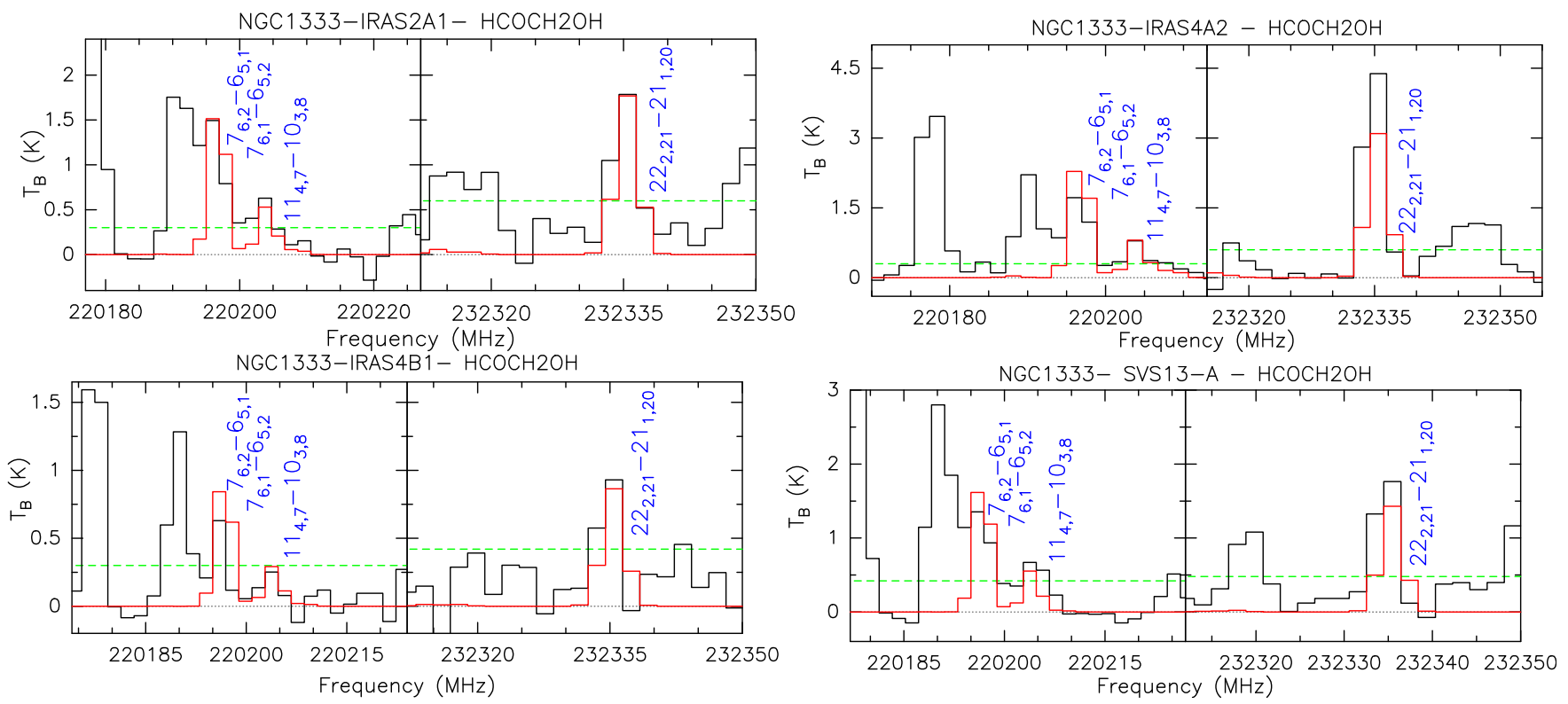

Fig. 1. Examples of glycolaldehyde emission lines (in $T_{\mathrm{B}}$ scale) observed in the $1.3 \mathrm{~mm}$ and $1.4 \mathrm{~mm}$ spectral windows (and at different upperlevel energy, $E_{\mathrm{u}}$ from $37 \mathrm{~K}$ to $135 \mathrm{~K}$ ) towards NGC 1333-IRAS2A1 (upper-left panel), NGC 1333-IRAS4A2 (upper-right panel), NGC 1333IRAS4B1 (lower-left panel), and NGC 1333-SVS13A (lower-right panel). The horizontal green dotted lines show the $3 \sigma$ level. In blue we mark the glycolaldehyde lines extracted from Tables A.1-A.4. The bright line at $220190 \mathrm{MHz}$ is due to $\mathrm{CH}_{3} \mathrm{OCHO}\left(17_{4,13}-16_{4,12}\right) \mathrm{A}$ (Maury et al. 2014; Belloche et al., in prep.). The red line shows the synthetic spectrum obtained with the GILDAS-Weeds package (Maret et al. 2011) and assuming the rotation diagram solutions (see Fig. 3).

\section{1. $\mathrm{HCOCH}_{2} \mathrm{OH}$ spatial distributions}

Figure 2 compares the spatial distribution of the glycolaldehyde emission (colour scale and green contours) with that of the $1.4 \mathrm{~mm}$ continuum (white contours; see Codella et al. 2014; Maury et al., in prep.) towards (from top to bottom) NGC 1333-IRAS2A1, NGC 1333-IRAS4A, NGC 1333IRAS4B1, and NGC 1333 SVS13-A. The glycolaldehyde distribution refers to emission lines in both the $1.3 \mathrm{~mm}$ and $1.4 \mathrm{~mm}$ spectral windows, i.e. the sum of the $7_{6,2}-6_{5,1}$ and $7_{6,1}-6_{5,2}$ lines $\left(220.2 \mathrm{GHz}, E_{\mathrm{u}}=37 \mathrm{~K}\right)$, and the $22_{2,21}-21_{1,20}$ line $(232.3 \mathrm{GHz}$, $\left.E_{\mathrm{u}}=135 \mathrm{~K}\right)$. Consistent with the results of Taquet et al. (2015), our images indicate that the glycolaldehyde emission is only marginally resolved. For each source, we derived the emission size of the brightest line at $1.3 \mathrm{~mm}$ shown in Fig. 2 by fitting an elliptical Gaussian in the $u v$ domain: we obtain about $0 \prime 25$ (IRAS2A1), 0"'4 (IRAS4A2, IRAS4B1), and 0".3 (SVS13-A), which implies that the glycolaldehyde emission is confined in the inner 100 au around each protostar. The glycolaldehyde emission peak is consistent, considered the angular resolution, with the continuum peaks. Our results confirm the hot-corino nature of NGC 1333-IRAS2A1 and NGC 1333-IRAS4A2, previously noted by Maury et al. (2014) and Taquet et al. (2015), and in addition supports the same nature for IRAS4B1 and SVS13-A (see Sect. 4.2 for the temperature measurements).

Finally, the image of the brightest glycolaldehyde emission (at $220.2 \mathrm{GHz}$ ) of the IRAS4-A1+A2 system, shows, in addition to the A 2 compact core, a tentative detection at $5 \sigma$ of a more extended emission. If significant, either this could trace part of the extended envelope hosting the two protostars; or it could be associated with the swept-up material associated with the two N-S outflows driven by the A1 and A2 protostars (Santangelo et al. 2015). In the latter case, this would be the first signature of glycolaldehyde emission from a protostellar outflow. However, note that this feature could be potentially contaminated by emission due to the $\mathrm{SO}^{17} \mathrm{O}\left(13_{2,12}-13_{1,13}\right)$ transition at $220196.75 \mathrm{MHz}$
$\left(E_{\mathrm{u}}=93 \mathrm{~K}, \log \left(A_{\mathrm{ij}} / \mathrm{s}^{-1}\right)=-3.8\right)$. We inspected the CALYPSO dataset searching for emission lines due to $\mathrm{SO}_{2}$ isotopologues. We have only a weak (less than $3 \sigma){ }^{34} \mathrm{SO}_{2}\left(4_{2,2}-\right.$ $\left.3_{1,3}\right)$ emission at $229.9 \mathrm{GHz}\left(E_{\mathrm{u}}=19 \mathrm{~K}, \log \left(A_{\mathrm{ij}} / \mathrm{s}^{-1}\right)=-4.1\right)$, but only around the NGC 1333 -IRAS4A2 protostar. In addition, again only IRAS4A2, we have a tentative $(\geq 3 \sigma)$ detection of the $\mathrm{SO}_{2}\left(11_{5,7}-12_{4,8}\right)$ line $\left(E_{\mathrm{u}}=122 \mathrm{~K}\right)$ at $229.3 \mathrm{GHz}$, and, not surprisingly, no detection of the $\mathrm{SO}_{2}\left(22_{7,15}-23_{6,18}\right)$ line at $219.3 \mathrm{GHz}\left(E_{\mathrm{u}}=352 \mathrm{~K}\right)$. In any case, the spectral resolution and sensitivity of the present dataset is not high enough to properly investigate this intriguing possibility, calling for further interferometric observations. The analysis presented in this paper only refers to the compact component.

\section{2. $\mathrm{HCOCH}_{2} \mathrm{OH}$ rotation diagrams}

Given that the glycolaldehyde collisional rates are not available in the literature, we derived excitation temperature and column densities by using a crude approach such as the classical rotational diagram, assuming a unique temperature, optically thin emission, and Local Thermodynamic Equilibrium (LTE) conditions. We applied a correction due to the beam filling factor using the sizes reported in Sect. 4.1. Under these assumptions, for a given transition the column density of the upper level, $N_{\text {up }}$, is related to the rotational temperature $T_{\text {rot }}$, as follows:

$N_{\mathrm{up}}=\frac{8 \pi k v^{2}}{h c^{3} A_{\mathrm{ul}} f f} \int T \mathrm{~d} v$

$\ln \frac{N_{\text {up }}}{g_{\text {up }}}=\ln N_{\text {tot }}-\ln Q\left(T_{\text {rot }}\right)-\frac{E_{\text {up }}}{k T_{\text {rot }}}$

where: $h$ and $k$ are the Planck and Boltzmann constants, respectively, $f f$ is the beam filling factor (derived using the sizes reported in Sect. 4.1), $g_{\text {up }}$ is the degeneracy of the upper level, 

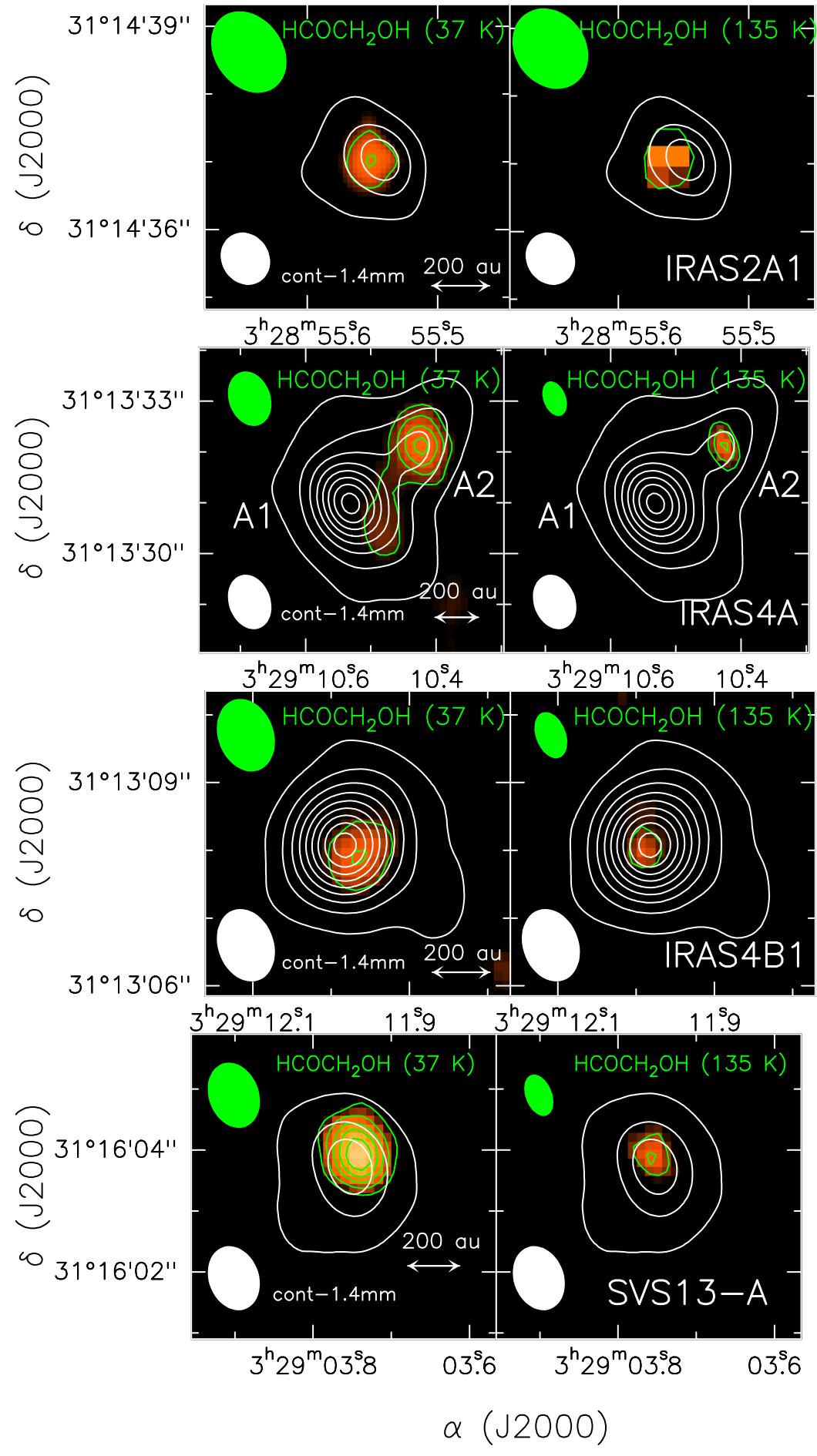

Fig. 2. Comparison between the $1.4 \mathrm{~mm}$ continuum (white contours) and the glycolaldehyde spatial distributions (colour scale and green contours) towards (from top to bottom) NGC 1333-IRAS2A1, NGC 1333IRAS4A, NGC 1333-IRAS4B1, and NGC 1333 SVS13A. For continuum, first contours and steps correspond to $5 \sigma\left(14 \mathrm{mJy}\right.$ beam $\left.^{-1}\right)$ and $10 \sigma$, respectively. The ellipses show the PdBI synthesised beam (HPBW) for the continuum (white, see Table 1) and glycolaldehyde images. The HPBWs for the glycolaldehyde images at $1.4 \mathrm{~mm}$ are: $0 \prime \prime 82 \times 0, \prime 70,32^{\circ}\left(\right.$ NGC 1333-IRAS2A1), $1^{\prime \prime} 08 \times 0$ 0 $0^{\prime \prime} 81$, $19^{\circ}$ (NGC 1333-IRAS4A), $1^{\prime \prime} .08 \times 00^{\prime \prime} 83,20^{\circ}$ (NGC 1333IRAS4B1), and $11^{\prime \prime} 06 \times 0$ 0'”8, $20^{\circ}$ (NGC 1333 SVS13-A). The HPBWs for the glycolaldehyde images at $1.3 \mathrm{~mm}$ are: $0 \prime \prime 82 \times 0, \prime 70,32^{\circ}\left(\right.$ NGC 1333-IRAS2A1), $0 \prime \prime 72 \times 00^{\prime \prime} .44$, $21^{\circ}$ (NGC 1333-IRAS4A), $0^{\prime \prime} .72 \times 00^{\prime \prime} 44,21^{\circ}$ (NGC 1333IRAS4B1), and $0^{\prime \prime} \cdot 71 \times 00^{\prime \prime} \cdot 43,21^{\circ}($ NGC 1333 SVS13-A). Left panels: glycolaldehyde distribution refers to the sum of the $7_{6,2}-6_{5,1}$ and $7_{6,1}-6_{5,2}$ emission with $(220.2 \mathrm{GHz}$, $E_{\mathrm{u}}=37 \mathrm{~K}$; see Tables A.1-A.4). First contours and steps correspond to $5 \sigma\left(90,90,75\right.$, and $55 \mathrm{mJy}^{\text {beam }}{ }^{-1}$ $\mathrm{km} \mathrm{s}^{-1}$ respectively for NGC 1333-IRAS2A1, NGC 1333IRAS4A, NGC 1333-IRAS4B1, and NGC 1333 SVS13-A) and $3 \sigma$, respectively. Right panels: glycolaldehyde distribution refers to the $22_{2,21}-21_{1,20}$ emission $\left(232.3 \mathrm{GHz}, E_{\mathrm{u}}=\right.$ $135 \mathrm{~K}$; see Tables 2-5). First contours and steps correspond to $5 \sigma\left(100,110,50\right.$, and $60 \mathrm{mJy}$ beam $^{-1} \mathrm{~km} \mathrm{~s}^{-1}$ respectively for NGC 1333-IRAS2A1, NGC 1333-IRAS4A, NGC 1333-IRAS4B1, and NGC 1333 SVS13-A) and $3 \sigma$, respectively.
$N_{\text {tot }}$ is the total column density, $Q\left(T_{\text {rot }}\right)$ is the partition function, and $E_{\text {up }}$ is the upper level energy. Figure 3 shows the rotation diagrams obtained using both the $1.3 \mathrm{~mm}$ (red points) and $1.4 \mathrm{~mm}$ (blue points) lines, while Table 2 reports the results, i.e. high temperatures, $T_{\text {rot }}$ between $115 \mathrm{~K}$ and $236 \mathrm{~K}$, and $N_{\text {tot }} \simeq 1-9 \times 10^{15} \mathrm{~cm}^{-2}$. The present data image, for the first time, a hot corino around SVS13-A and NGC 1333-IRAS4B1. These findings confirm that the glycolaldehyde gas is present in the region with a radius less than 100 au where the temperature is high enough to sublimate the dust mantles. Thus, either glycolaldehyde is directly released from mantle sublimation or formed through gas-phase reactions using simpler species directly formed on the mantles and successively evaporated due to thermal heating. Note that the sources where we report a detection of glycolaldehyde are those with the highest number of spectral lines of COMs (e.g. $\mathrm{HCOOCH}_{3}$ ) detected above $6 \sigma$ in the $1.3 \mathrm{~mm}$ and $1.4 \mathrm{~mm}$ WideX spectra of the CALYPSO subsample of Perseus sources (Belloche et al., in prep.). In the case of IRAS2A1 and IRAS4A2, the estimated rotational temperatures and column densities are well in agreement (after applying correctly the beam filling factor scaling) with what was previously reported by Taquet et al. (2015) and Coutens et al. (2015) using different datasets on similar spatial scales, thus confirming the goodness of the present line identification. On the other hand, 

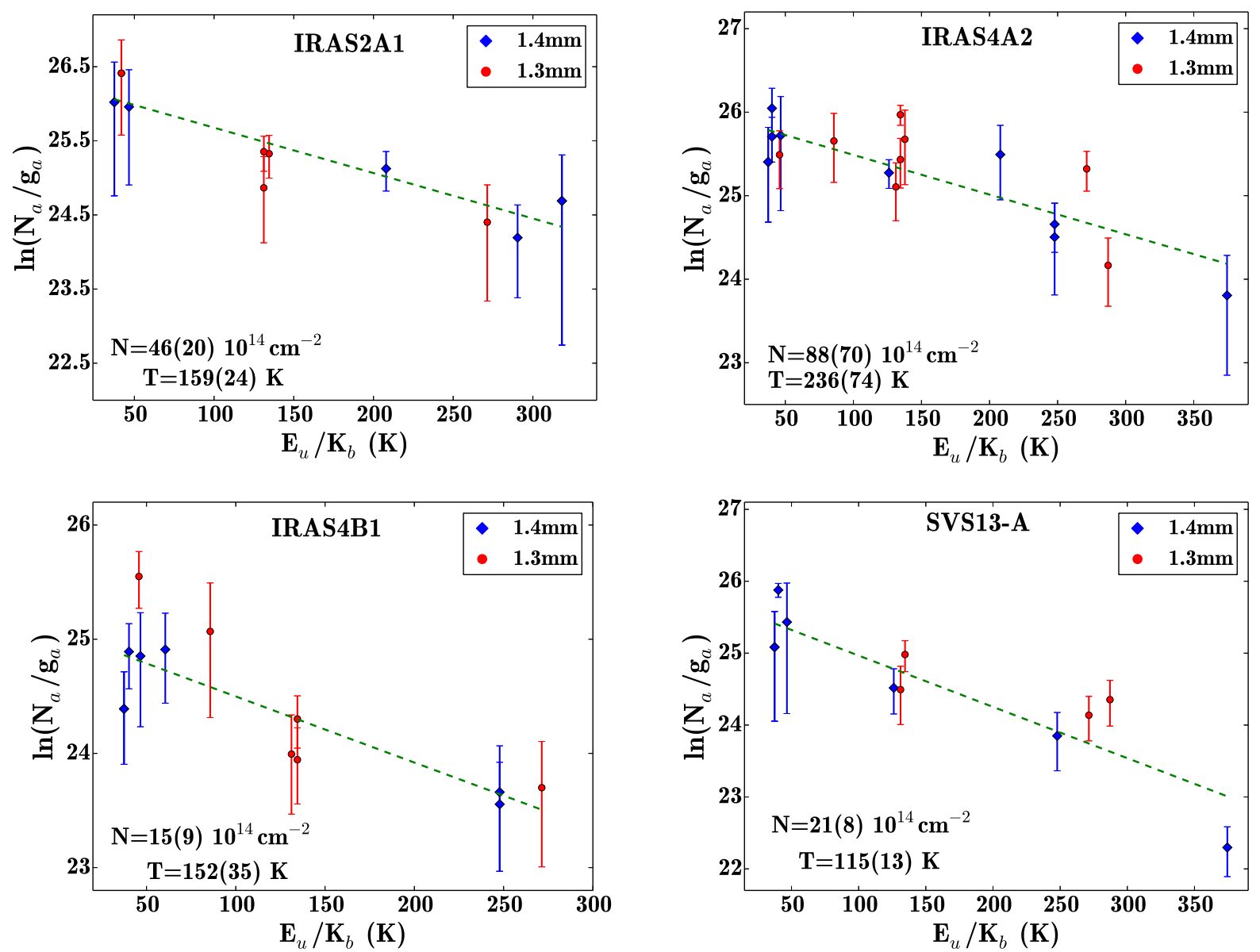

Fig. 3. Rotation diagram for the glycolaldehyde transitions reported in Table 2 for NGC 1333-IRAS2A (upper-left panel), NGC 1333-IRAS4A2 (upper-right panel), NGC 1333-IRAS4B1 (lower-left panel), and NGC 133-SVS13A (lower-right panel). The line intensities have been corrected for beam dilution using the sizes derived in Sect. 4.1. The parameters $N_{\text {up }}, g_{\text {up }}$, and $E_{\text {up }}$ are respectively the column density, the degeneracy, and the energy of the upper level. The error bars on $\ln \left(N_{\text {up }} / g_{\text {up }}\right)$ are given by the vertical bar of the symbols. Red and blue points refer to the lines detected in the $1.3 \mathrm{~mm}$ and $1.4 \mathrm{~mm}$ spectral bands, respectively. The dashed lines represent the best fits.

the derived $N_{\text {tot }}$ are lower than what Jørgensen et al. (2012, 2016) towards the IRAS $16293-2422$ binary $\left(3-4 \times 10^{16} \mathrm{~cm}^{-2}\right)$ found, in Ophiuchus, on smaller $(\leq 60 \mathrm{au})$ spatial scales. The sources where we report a detection of glycolaldehyde are those with the highest number of spectral lines of complex organic molecules detected above 6 sigma in the $1.3 \mathrm{~mm}$ and $1.4 \mathrm{~mm}$ WIDEX spectra of the CALYPSO subsample of Perseus sources (Belloche et al., in prep.).

The rotation diagram solutions have been used to create synthetic spectra (see the red lines in Figs. 1 and A.1 to A.9) to strengthen the detection using the GILDAS-Weeds package (Maret et al. 2011). We assumed a background temperature of $2.73 \mathrm{~K}$. In principle, for sources with a strong continuum such as NGC 1333-IRAS4B1, the column density obtained using the rotational diagram method can be underestimated, converting the measurement in a lower limit. The synthetic intensities are not systematically over- or under-estimating the observed lines, confirming that the temperatures derived from the rotation diagrams are good approximations. Note that all the detected lines are optically thin. However, even though the rotational diagrams show reasonable agreement between measurements and the single temperature model, it is worth to point out here some shortcomings of our models in reproducing the observed spectra. While we do not find large discrepancies, the spectral resolution of our observations is relatively low and the spectra very rich of emission lines (especially of the four sources where we do detect glycolaldehyde line). This implies that in some cases the lines may be contamined by emission from other molecules. Figures A.1, A.3, A.5, and A.7 shows the lines selected for the analysis. To be thorough, Figs. A.2, A.4, A.6, and A.8 show all the lines excluded from the present analysis because, although they are consistent with the observed spectrum, the blends with other species prevents a firm identification of the line. Note that, in a few cases, our simple LTE model predicts higher fluxes than observed; a special case is represented by the $20_{2,18}-19_{3,17}$ line at $220463 \mathrm{MHz}$ observed towards NGC 1333-IRAS4B1, -IRAS2A1, IRAS4A2, and-SVS13A (see Fig. A.9), and conservatively excluded from the LTE analysis falling at the edge of the observed WideX band. Beside this line, only in 2 cases (over a grand total of about 90 lines, counting also those not used here because of blending) the discrepancy reaches a factor 3: the $22_{1,21}-21_{2,20}$ line at $\sim 232286 \mathrm{MHz}$ in IRAS2A1 (see Fig. A.2), and the $9_{5,5}-8_{4,4}$ line at $\sim 217626 \mathrm{MHz}$ in SVS13-A (see Fig. A.8). The difference is even less once considered both the errors associated with the LTE fit (see Table 2) and the errors on the observed line peak intensity. Indeed, for lines close to the detection limit the systematic discrepancy could be understood in terms of the noise fluctuations and the uncertainty of the continuum subtraction in a region of the spectrum very rich of lines. We also note that deviations from the simplifying 


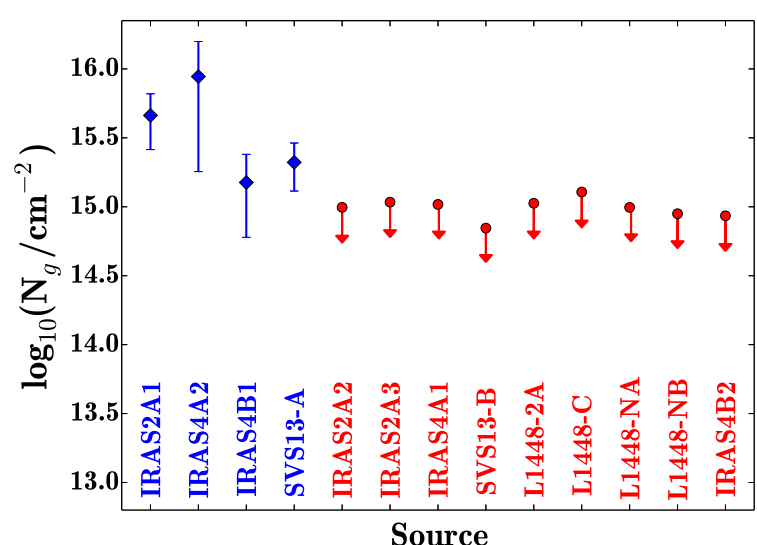

Fig. 4. Distribution of the glycolaldehyde column density $\left(N_{\text {tot }}\right)$ derived towards the selected sample (see Table 1). Red arrows are for upper limits.

assumptions of single temperature LTE, non thermal excitation and absorption may be the cause of some of the discrepancies. Obtaining higher sensitivity and higher spectral and spatial resolution observations over a broader frequency range, together with a full spectrum modeling, will help deriving more accurate estimates of the gas parameters. Nonetheless, eventually the intrinsic linewidth, the source chemical complexity and richness of the spectra and the source physical complexity will always limit the ability to accurately identify and measure individual lines. Overall, our simple LTE analysis and comparison with the spectra show that, while not perfect, it is possible to estimate a temperature and column density representative of the bulk of the molecular emission.

\section{3. $\mathrm{HCOCH}_{2} \mathrm{OH}$ occurrence around protostars}

Figure 4 compares the column densities measured towards the four sources detected in glycolaldehyde with the upper limits on $N_{\text {tot }}$ derived for the 9 objects in Perseus where glycolaldehyde has not been revealed. Assuming a typical temperature of $150 \mathrm{~K}$, a size of 0'.4 (see Sect. 4.1), and considering the $3 \sigma$ values of the spectra where no glycolaldehyde emission has been found, the typical upper limit is $\leq 10^{15} \mathrm{~cm}^{-2}$, i.e. one order of magnitude less than what found in IRAS2A1, IRAS4A2, IRAS4B1, and SVS13-A. These measurements support that these four protostars show a significantly higher column density of glycolaldehyde molecules in the gas phase. However, this may not reflect directly a difference in glycolaldehyde abundance.

To estimate the possible enhancement (or deficit) of gas phase glycolaldehyde abundance in the sources in our sample we need to compare with an estimate of the total gas column density in each source. As a proxy for the total column density towards each source we use the peak continuum flux at $1.4 \mathrm{~mm}$. The millimetre continuum flux in these sources is dominated by the thermal emission from dust grains. The exact conversion of the millimeter flux into total gas column density depends on three very uncertain factors: the dust properties (defining the dust opacity per gram of dust), the dust density and temperature structure, and the gas to dust ratio. Comparing the observed glycolaldehyde column densities with the continuum peak flux will allow to search for systematic abundance variations in our sample only under the assumption that the sources in our sample have uniform properties. While this is expected, since our sources are an homogeneous set of protostars in the same star forming region,

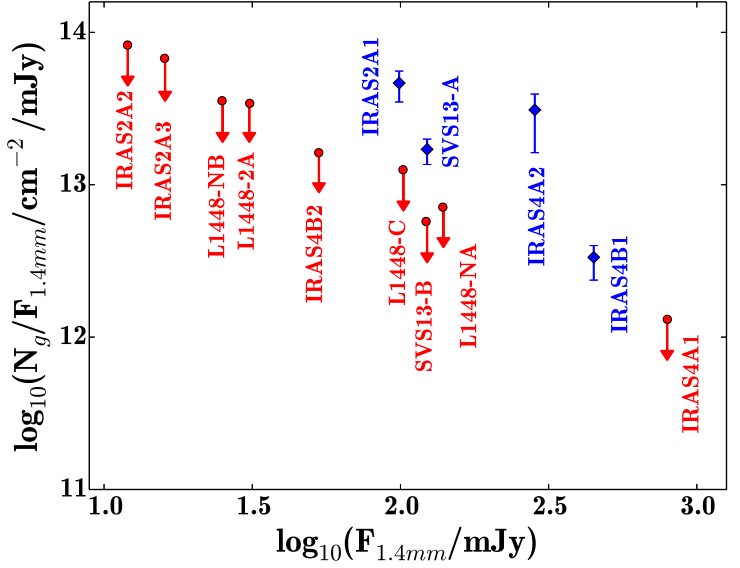

Fig. 5. Ratio between the glycolaldehyde column density $\left(N_{\text {tot }}\right)$ and the peak flux of the $1.4 \mathrm{~mm}$ continuum emission $\left(F_{1.4 \mathrm{~mm}}\right.$; Maury et al., in prep.) versus the $1.4 \mathrm{~mm}$ peak flux. Red arrows are for sources where glycolaldehyde has not been detected.

in the future this analysis will have to be refined using more extensive observations and more accurate estimates of the total gas column density in each source.

Figure 5 shows the ratio between the glycolaldehyde column density, $N_{\text {tot }}$, and the continuum peak flux at $1.4 \mathrm{~mm}\left(F_{1.4} \mathrm{~mm}\right.$, from Maury et al., in prep.) against the value of the $1.4 \mathrm{~mm}$ peak flux itself. As mentioned above, if we assume that the molecular hydrogen column density scales with the dust peak flux density, then the plot in Fig. 5 shows that the gas phase abundance of glycolaldehyde relative to molecular hydrogen has a spread of a factor of about 10 among the sources with glycolaldehyde detections. Even if the non-detection of sources with $F_{1.4} \mathrm{~mm}$ less than $100 \mathrm{mJy}$ might suggest that a detection requires bright continuum emission, a bright continuum source is not necessarily associated with glycolaldehyde emission. A typical example is given by the IRAS4-A1+A2 system, where it is only the object showing weaker continuum emission that is detected in glycolaldehyde (see Fig. 2). If we consider the sources with continuum peak flux above $\sim 100 \mathrm{mJy} / \mathrm{beam}$, then the average ratios for sources with detected glycolaldehyde are a factor of $\sim 10$ higher than the sources with upper limits. This suggests that the detected sources may have a significantly higher gas phase abundance of glycolaldehyde compared with the non-detected sources.

The glycolaldehyde detection indicates that the gas reached a temperature of at least $100 \mathrm{~K}$; at these high temperatures the gas is enriched chemically by the release of molecules directly from the dust mantles and/or by the overcome of the activation barrier of gas phase reactions using what was released from the icy dust mantles. It is reasonable to assume that the temperature increases with the source luminosity, which depends on the protostellar mass and on the source accretion rate, which in turn is related to the evolutionary stage. However, only three sources where glycolaldehyde has been detected can be associated with a measurement of the internal luminosity hampering a reliable check for a trend (see Table 1). In addition, our $1.4 \mathrm{~mm}$ fluxes do not allow us to derive a direct measurement of the protostellar mass. Trying to shed light on this aspect, we note that in the case of the SVS13-A+B system, glycolaldehyde is only detected towards the more evolved SVS13-A source, with $L_{\text {int }}=24.5 L_{\odot}$, whereas the younger object SVS13-B, with $L_{\text {int }}=1-2 L_{\odot}$. does not show evidence of an enriched chemistry. Therefore, at least 
in this case, the lack of a detected hot-corino in SVS13-B suggests that the protostar had not enough time to warm a portion of gas large/bright enough to be detectable. Interestingly, Santangelo et al. (2015), using the $\mathrm{SiO}$ and $\mathrm{CO}$ jet properties imaged in IRAS4-A1+A2 system, proposed that the chemical richness observed towards IRAS4-A2 (and not towards IRAS4-A1), is due to a later evolutionary stage, inferred from the jet properties driven by the two objects. Nevertheless, it is clear that a more systematic comparative study of sources in different evolutionary phases is required to reach a firm conclusion: based only on a few sources (e.g. SVS13-A+B), it is very difficult to tell whether the primary factor leading to the detection of glycolaldehyde emission is evolution (e.g. low value of $L_{\text {submm }} / L_{\text {int }}$ ) or accretion luminosity (high $L_{\text {int }}$ ) or environment (e.g. NGC 1333 vs. L1448). These findings call for further analysis of the chemical content of the protostellar environments of the whole CALYPSO sample, using all the emission due to all the COMs and not only to glycolaldehyde, which will be presented in a forthcoming paper (Belloche et al., in prep.).

\section{Conclusions}

We report a survey of glycolaldehyde emission towards a sample of Class 0 protostars located in the L1448 and NGC 1333 star forming regions, in Perseus. The analysis has been performed in the framework of the IRAM PdBI CALYPSO survey. The main findings can be summarised as follows:

1. We found glycolaldehyde towards 3 Class 0 objects (NGC 1333-IRAS2A1， NGC 1333-IRAS4A2， NGC 1333IRA4B1) and 1 Class I source (SVS13-A). Once excluded the continuum peaks whose nature is still debated, this results in a detection rate of $\sim 36 \%$. The NGC 1333 star forming region is particularly rich in glycolaldehyde, with a definitely higher rate of occurrence: up to $60 \%$.

2. The glycolaldehyde emission is compact and coincident with the continuum peak of each detected source. The brightest lines indicate typical sizes of $00^{\prime \prime} 4$ showing that the glycolaldehyde emission is tracing the inner 100 au around the protostar. We detected a large (up to 18) number of lines, covering a large range of upper-level energy, with $E_{\mathrm{u}}$ from $37 \mathrm{~K}$ up to $375 \mathrm{~K}$, and indicating high temperatures, $T_{\text {rot }}$ between $100 \mathrm{~K}$ and $200 \mathrm{~K}$. We confirm and extend earlier results towards IRAS2A1 and IRAS4A2, and reveal for the first time the presence of hot corinos around NGC 1333IRAS4B1 and SVS13-A. The total column densities of glycolaldehyde in the detected sources are $\simeq 1-9 \times 10^{15} \mathrm{~cm}^{-2}$.

3 . We suggest that the four hot corinos imaged in glycolaldehyde in NGC 1333 are associated with a significantly higher gas phase abundance of glycolaldehyde with respect to the rest of the sample. The SVS13-A+B and IRAS4-A1+A2 systems suggest that the detection of glycolaldehyde emission is due to the source evolution: the chemical richness highlights those protostars evolved enough to warm a bright region of $\sim 100$ au at temperatures larger than $100 \mathrm{~K}$. However, the present sample does not allow us to reach a firm conclusion whether the glycolaldehyde detection is always a question of evolution or accretion luminosity and/or if it depends on the environment hosting the protostars.

Our initial results can be confirmed and extended with more sensitive observations of a larger sample of objects. In particular, more accurate determinations of the possible variations of glycolaldehyde abundance in gas phase will be a powerful probe of the chemical evolution of protostars and may confirm the present results.

Acknowledgements. We are very grateful to all the IRAM staff, whose dedication allowed us to carry out the CALYPSO project. This work was partly supported by the PRIN INAF 2012 - JEDI and by the Italian Ministero dell'Istruzione, Università e Ricerca through the grant Progetti Premiali 2012 - iALMA (CUP C52I13000140001). A.M. is supported by the MagneticYSOs, grant agreement No. 679937. C.C., L.T., and A.M. have been partially supported by the Gothenburg Centre for Advanced Studies in Science and Technology as part of the GoCAS program Origins of Habitable Planets. The research leading to these results has received funding from the European Community's Seventh Framework Programme (FP7/2007-2013/) under grant agreements No. 229517 (ESO COFUND) and No. 291294 (ERC ORISTARS), and from the French Agence Nationale de la Recherche (ANR), under reference ANR-12-JS05-0005.

\section{References}

André, P., Ward-Thompson, D., \& Barsony, M. 1993, ApJ, 406, 122

André, P., Ward-Thompson, D., \& Barsony, M. 2000, Protostars and Planets IV, eds. V. Mannings, A. P. Boss, \& S. S. Russell (Tucson: University of Arizona Press), 59

André, P., Men'shchikov, A., Bontemps, S., et al. 2010, A\&A, 518, L102

Anderl, S., Maret, S., Cabrit, S., et al. 2016, A\&A, 591, A3

Bachiller, R., Guilloteau, S., Gueth, F., et al. 1998, A\&A, 339, L49

Beltrán, M. T., Codella, C., Viti, S., Neri, R., \& Cesaroni, R. 2009, ApJ, 690, L93

Butler,, R. A. H., De Lucia,, F. C., Petkie, D. T., et al. 2001, ApJS, 134, 319

Calcutt, H., Viti, S., Codella, C., et al. 2014, MNRAS, 443, 3157

Carroll, P. B., Drouin, B. J., \& Widicus Weaver, S. L. 2010, ApJ, 723, 845

Ceccarelli, C., Caselli, P., Herbst, E., Tielens, A. G. G. M., \& Caux, E. 2007, Protostars and Planets V, eds. V. B. Reipurth, D. Jewit \& K. Keil (Tucson: University of Arizona Press), 47

Chen, X., Launhardt, R., \& Henning, T. 2009, ApJ, 691, 1729

Choi, M., Kang, M., Tatematsu, K., Lee,, J.-E., \& Park, G. 2011, PASP, 63, 1281

Codella, C., Benedettini, M., Beltrán,, M. T., et al. 2009, A\&A, 507, L25

Codella, C., Maury, A., Gueth, F., et al. 2014, A\&A, 563, L3

Coutens, A., Persson, M. V., Jørgensen, J. K., Wampfler, S. F., \& Lykke, J. M. 2015, A\&A, 576, A5

Dunham, M. M., Crapsi, A., Evans II, N. J., et al. 2008, ApJS, 179, 249

Halfen, D. T., Apponi, A. J., Woolf, N., Polt, R., \& Ziurys, L. M. 2006, ApJ, 639, 237

Herbst, E., \& van Dishoeck, E. F. 2009, ARA\&A, 47, 427

Hirota, T., Bushimata, T., Choi, Y. K., et al. 2008, PASJ, 60, 37

Hirota, T., Honma, N., Imai, H., et al. 2011, PASJ, 63, 1

Hollis, J. M., Lovas, F. J., \& Jewell, P. R. 2000, ApJ, 540, L107

Hollis, J. M., Jewell, P. R., Lovas, F. J., \& Remijan, A. 2004, ApJ, 613, L45

Jalbout, A. F., Abrell, L., Adamowicz, L., et al. 2007, Astrobiology, 7, 433

Jørgensen, J. K., Favre, C., Bisschop, S. E., et al. 2012, ApJ, 757, L4

Jørgensen, J. K., van der Wiel, M. H. D., Coutens, A., et al. 2016, A\&A, 595, A117

Lada, C. J. 1987, in Star Forming Regions, eds. M. Peimbert, \& J. Jugaku, IAU Symp., 115, 1

Lefloch, B., Castets, A., Cernicharo, J., Langer, W. D., \& Zylka, R. 1998, A\&A, 334, 269

Looney, L. W., Mundy, L. G., \& Welch, W. J. 2000, ApJ, 529, L477

Maret, S., Hily-Blant, P., Pety, J., Bardeau, S., \& Reynier, E. 2011, A\&A, 526, A47

Marstokk, K.-M., \& Møllendal, H. 1970a, J. Mol. Struct., 5, 205

Marstokk, K.-M., \& Møllendal, H. 1970b, J. Mol. Struct., 16, 259

Maury, A. J., Belloche, A., André Ph., et al. 2014, A\&A, 563, L2

Pickett, H. M., Poynter, R. L., Cohen, E. A., et al. 1998, J. Quant. Spectr. Rad. Transf., 60, 883

Requena-Torres, M. A., Martín-Pintado, J., Martín, S., \& Morris , M. R. 2008, ApJ, 672, 352

Sakai, N., Sakai, T., Hirota, T., et al. 2014, Nature, 507, 78

Santangelo, G., Codella, C., Cabrit, S., et al. 2015, A\&A, 584, A216

Taquet, V., López-Sepulcre, A., Ceccarelli, C., et al. 2015, ApJ, 804, 81

Tobin, J. J., Looney, L. W., Li, Z.-Y., et al. 2016, ApJ, 818, 73

Weber, A. L. 1998, Origins of Life and Evolution of the Biosphere, 28, 259

Widicus Weaver, S. L., Butler, R. A., H., Drouin, B. J., et al. 2005, ApJS, 158, 188 


\section{Appendix A: Observed $\mathrm{HCOCH}_{2} \mathrm{OH}$ emission lines}

Tables A.1 to A.4 report, for the 4 sources where glycolaldehyde emission has been detected (IRAS2A, SVS13-A, IRAS4A2, and IRAS4B), the results of the Gaussian fit for all the detected lines. In particular, we first list the spectral parameters (frequency, $v$, upper level energy, $E_{\mathrm{u}}$, and the $S \mu^{2}$ product, all taken from the Jet Propulsion Laboratory database; Pickett et al. 1998), followed by the line parameters (in $T_{\mathrm{MB}}$ scale), namely: the integrated emission $\left(\int T \mathrm{~d} v\right)$, both peak line velocities $\left(V_{\text {peak }}\right)$ and intensities $\left(T_{\text {peak }}\right)$, linewidth (FWHM), and rms noise. Figures A.1, A.3, A.5, and A.7 show the lines detected and used for the analysis, Figs. A.2, A.4, A.6, and A.8 show the lines excluded from the analysis given the severe blending with other emission lines, while Fig. A.9 is for a line which has not been used because it is located at the edge of the WideX bandwidth.
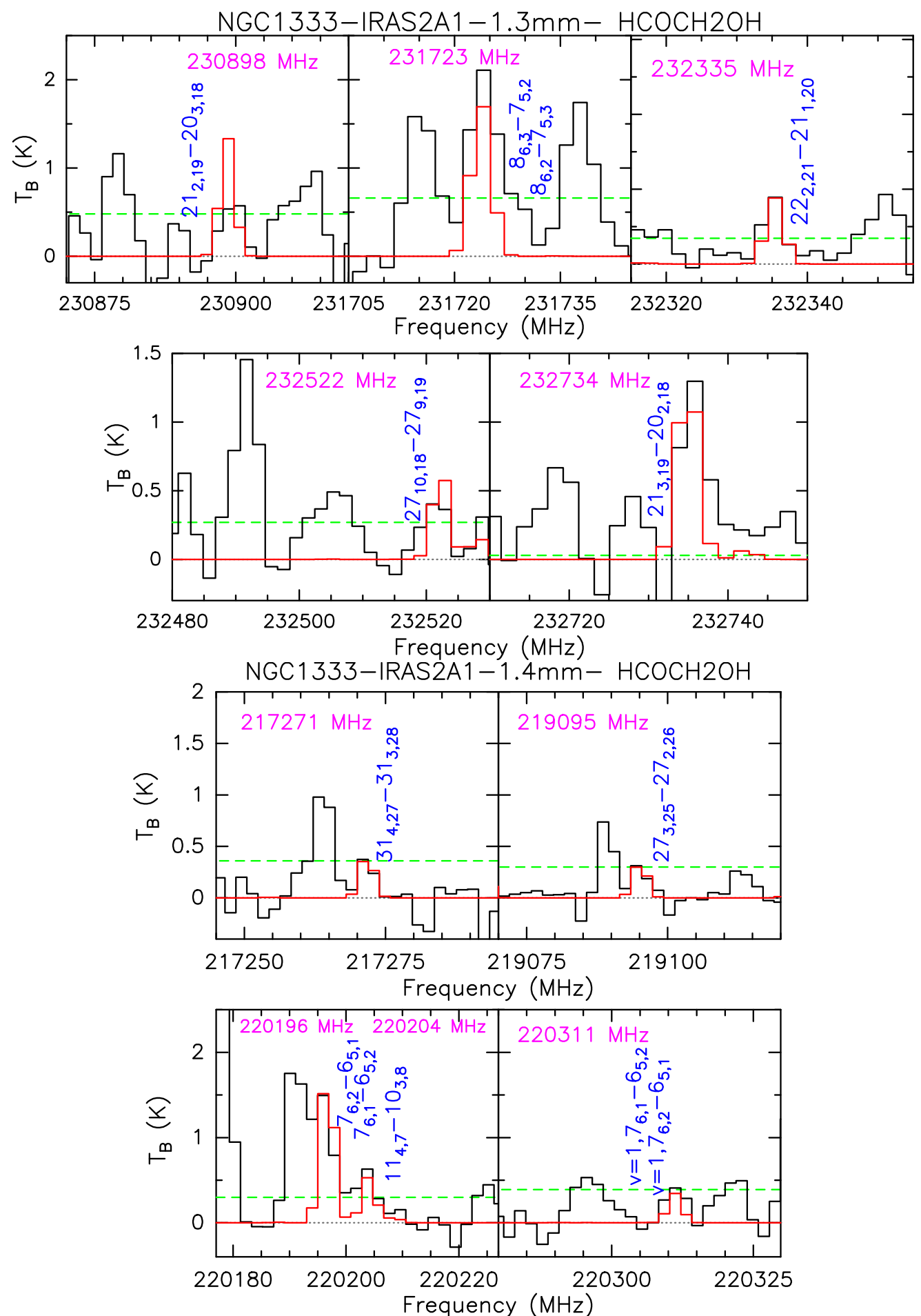

Fig. A.1. Glycolaldehyde emission lines (in $T_{\mathrm{B}}$ scale) detected in the $1.3 \mathrm{~mm}$ and $1.4 \mathrm{~mm}$ spectral windows towards NGC 1333-IRAS2A1 and used to perform the LTE analysis. The horizontal green dotted lines show the $3 \sigma$ level. In blue we mark the glycolaldehyde lines extracted from Table A.1. The red line shows the synthetic spectrum obtained with the GILDAS-Weeds package (Maret et al. 2011) and assuming the rotation diagram solutions (see Fig. 3). 

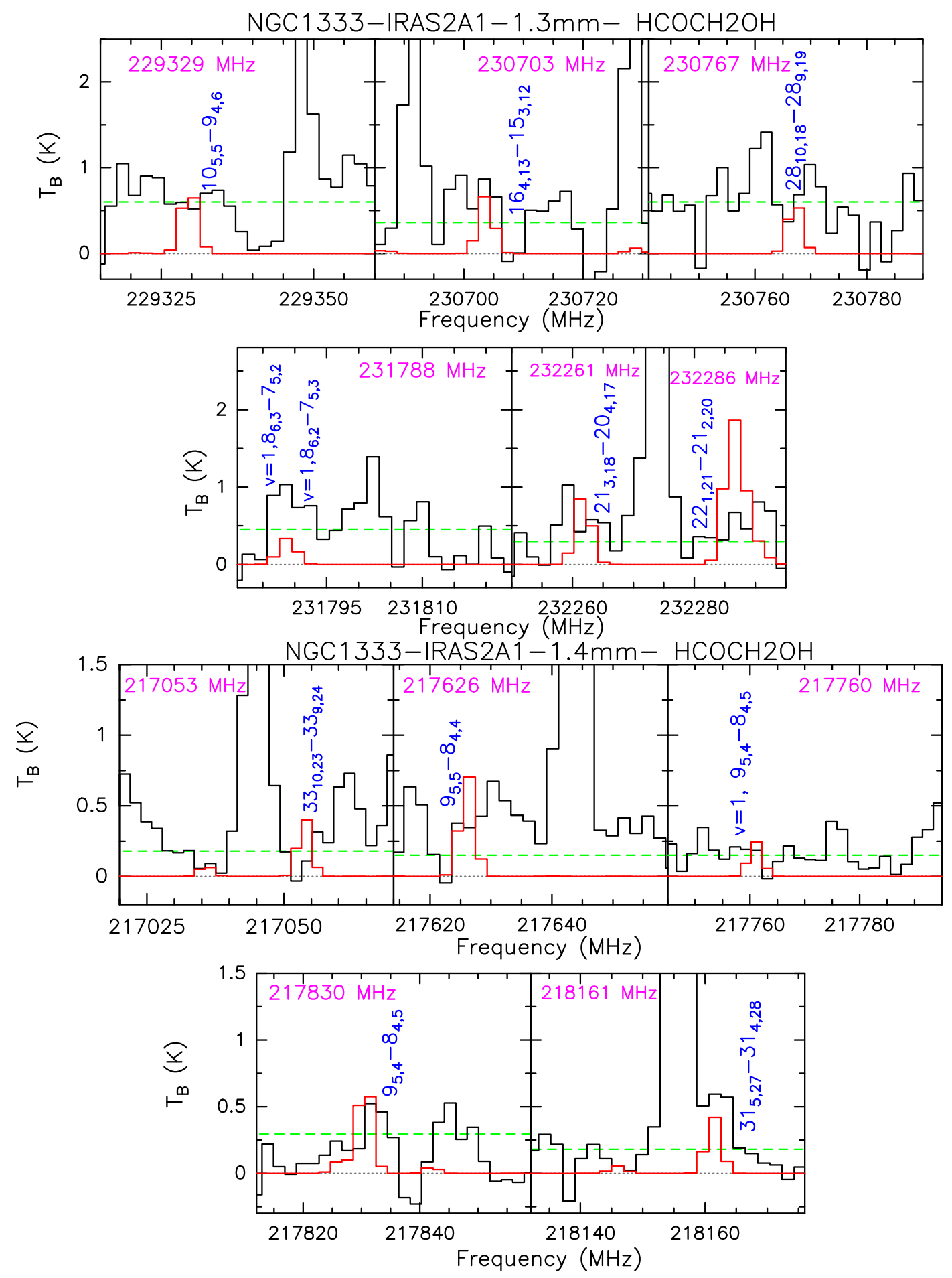

Fig. A.2. Glycolaldehyde emission lines (in $T_{\mathrm{B}}$ scale) observed in the $1.3 \mathrm{~mm}$ and $1.4 \mathrm{~mm}$ spectral windows towards NGC 1333-IRAS2A1 and excluded from the LTE analysis because of severe blending with other emission lines. The horizontal green dotted lines show the $3 \sigma$ level. In blue we mark the glycolaldehyde transitions. The red line shows the synthetic spectrum obtained with the GILDAS-Weeds package (Maret et al. 2011) and assuming the rotation diagram solutions (see Fig. 3). 
M. De Simone et al.: Glycolaldehyde in Perseus young solar analogs

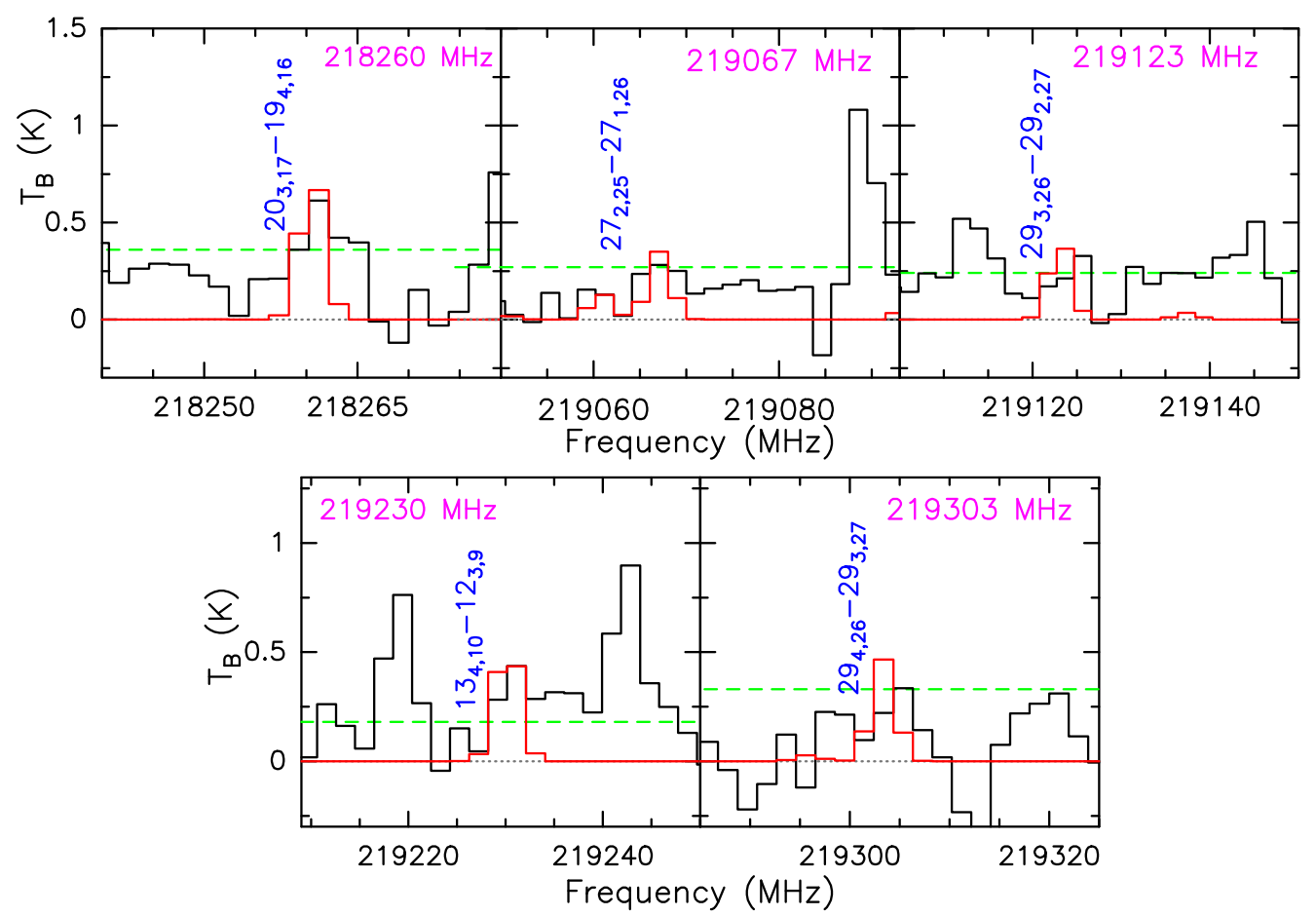

Fig. A.2. continued. 

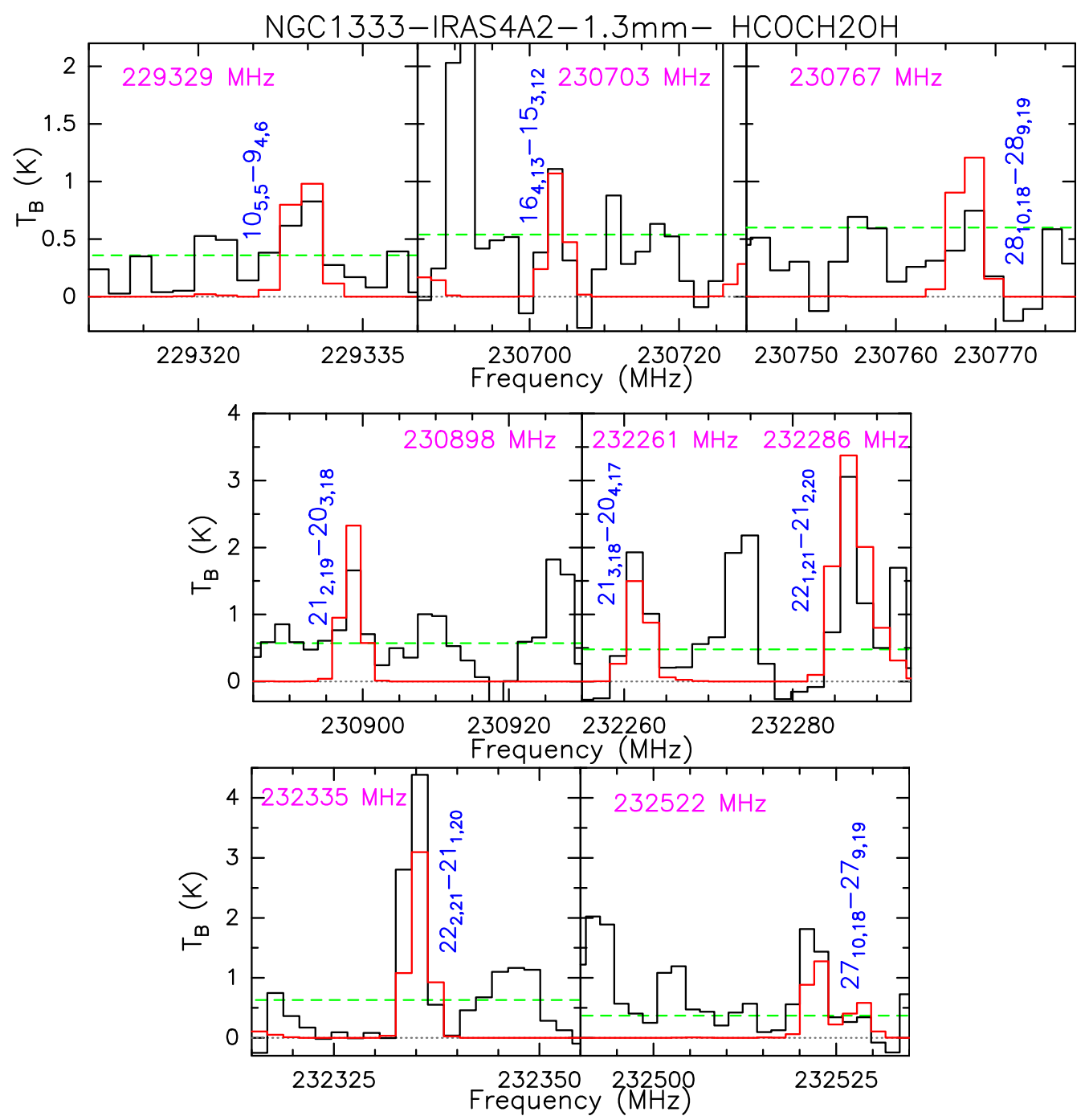

Fig. A.3. Glycolaldehyde emission lines (in $T_{\mathrm{B}}$ scale) detected in the $1.3 \mathrm{~mm}$ and $1.4 \mathrm{~mm}$ spectral windows towards NGC 1333-IRAS4A2 and used to perform the LTE analysis. The horizontal green dotted lines show the $3 \sigma$ level. In blue we mark the glycolaldehyde lines extracted from Table A.2. The red line shows the synthetic spectrum obtained with the GILDAS-Weeds package (Maret et al. 2011) and assuming the rotation diagram solutions (see Fig. 3). 
M. De Simone et al.: Glycolaldehyde in Perseus young solar analogs
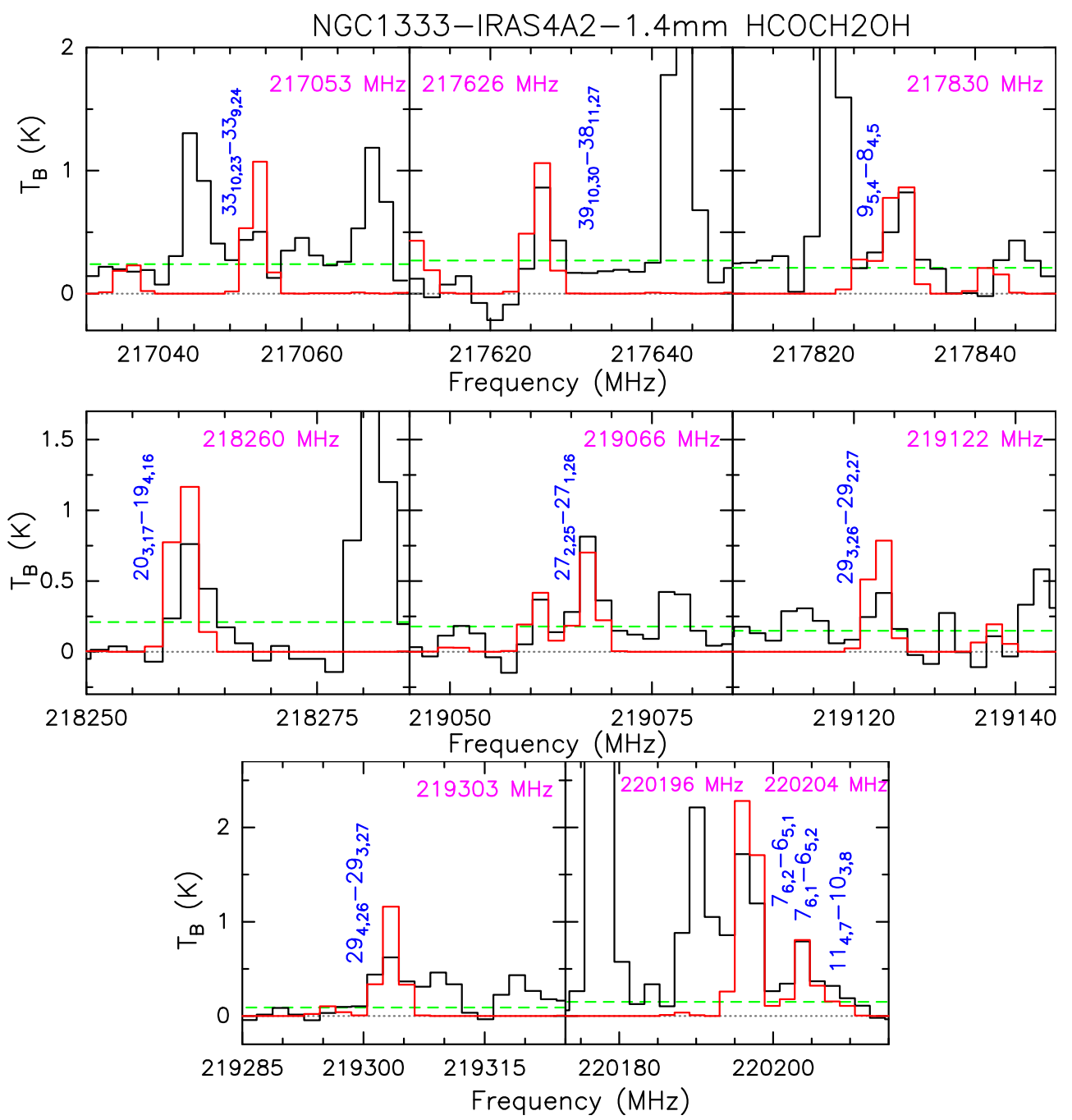

Fig. A.3. continued. 

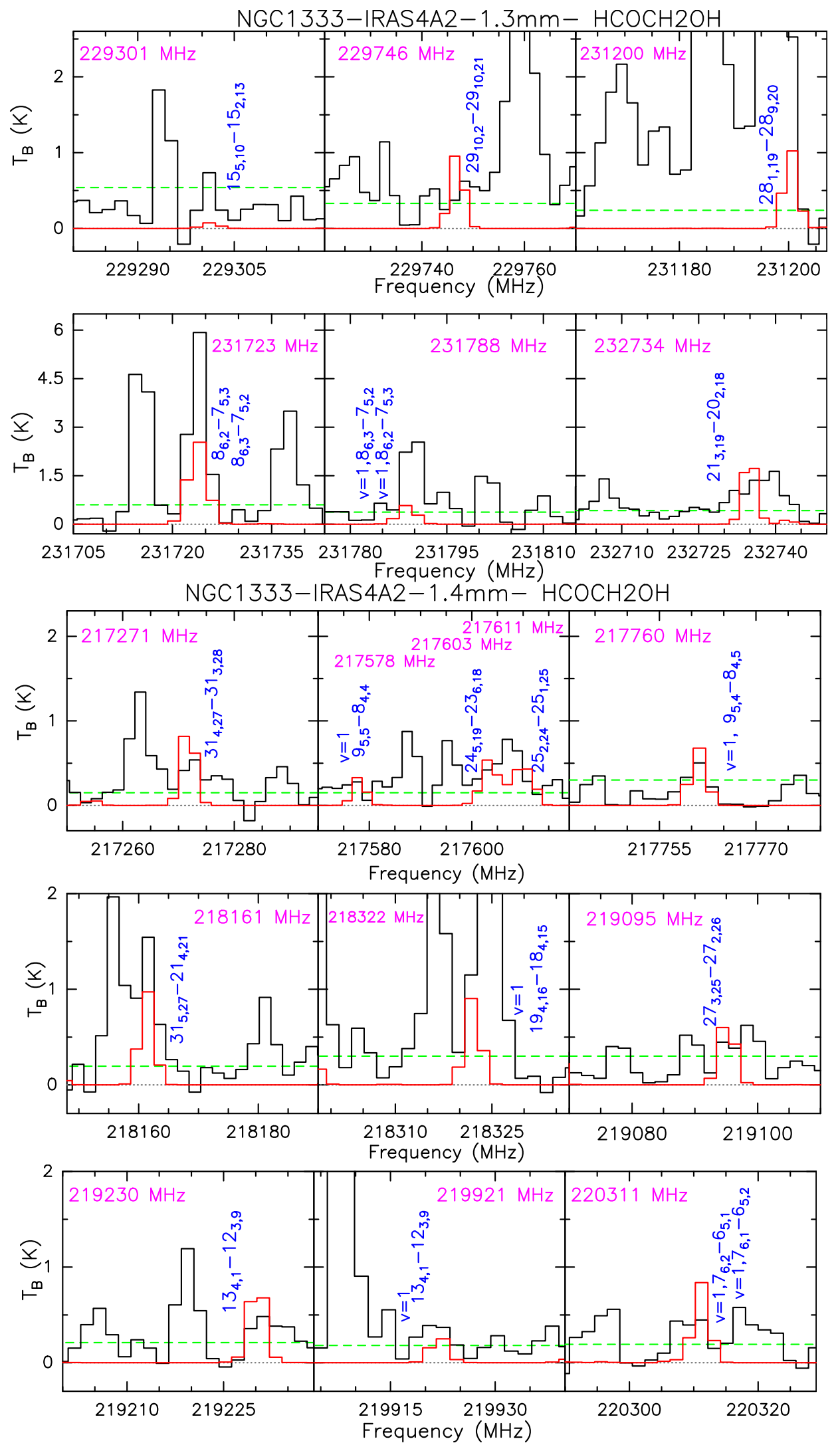

Fig. A.4. Glycolaldehyde emission lines (in $T_{\mathrm{B}}$ scale) observed in the $1.3 \mathrm{~mm}$ and $1.4 \mathrm{~mm}$ spectral windows towards NGC 1333-IRAS4A2 and excluded from the LTE analysis because of severe blending with other emission lines. The horizontal green dotted lines show the $3 \sigma$ level. In blue we mark the glycolaldehyde transitions. The red line shows the synthetic spectrum obtained with the GILDAS-Weeds package (Maret et al. 2011) and assuming the rotation diagram solutions (see Fig. 3). 
M. De Simone et al.: Glycolaldehyde in Perseus young solar analogs
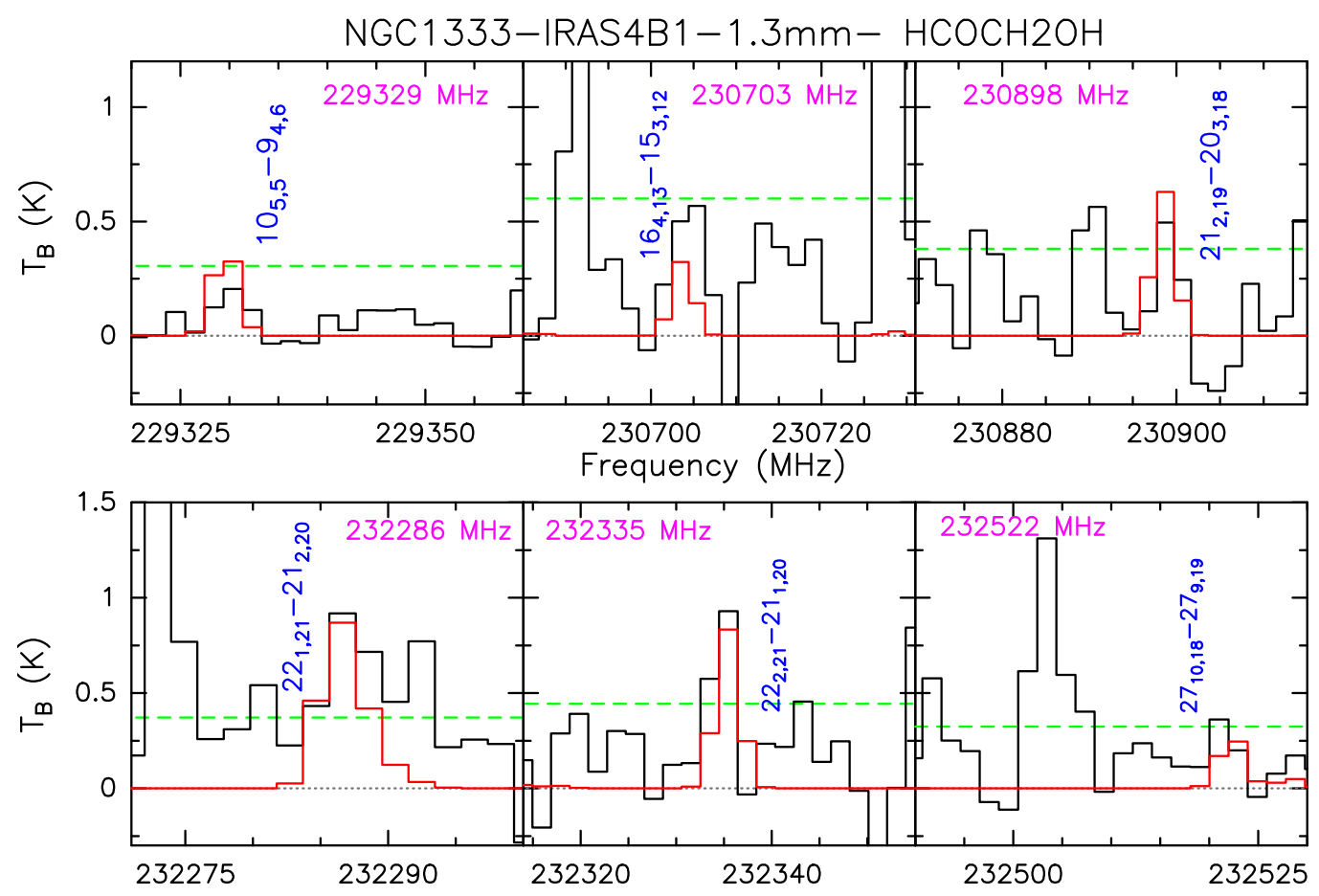

Frequency $(\mathrm{MHz})$
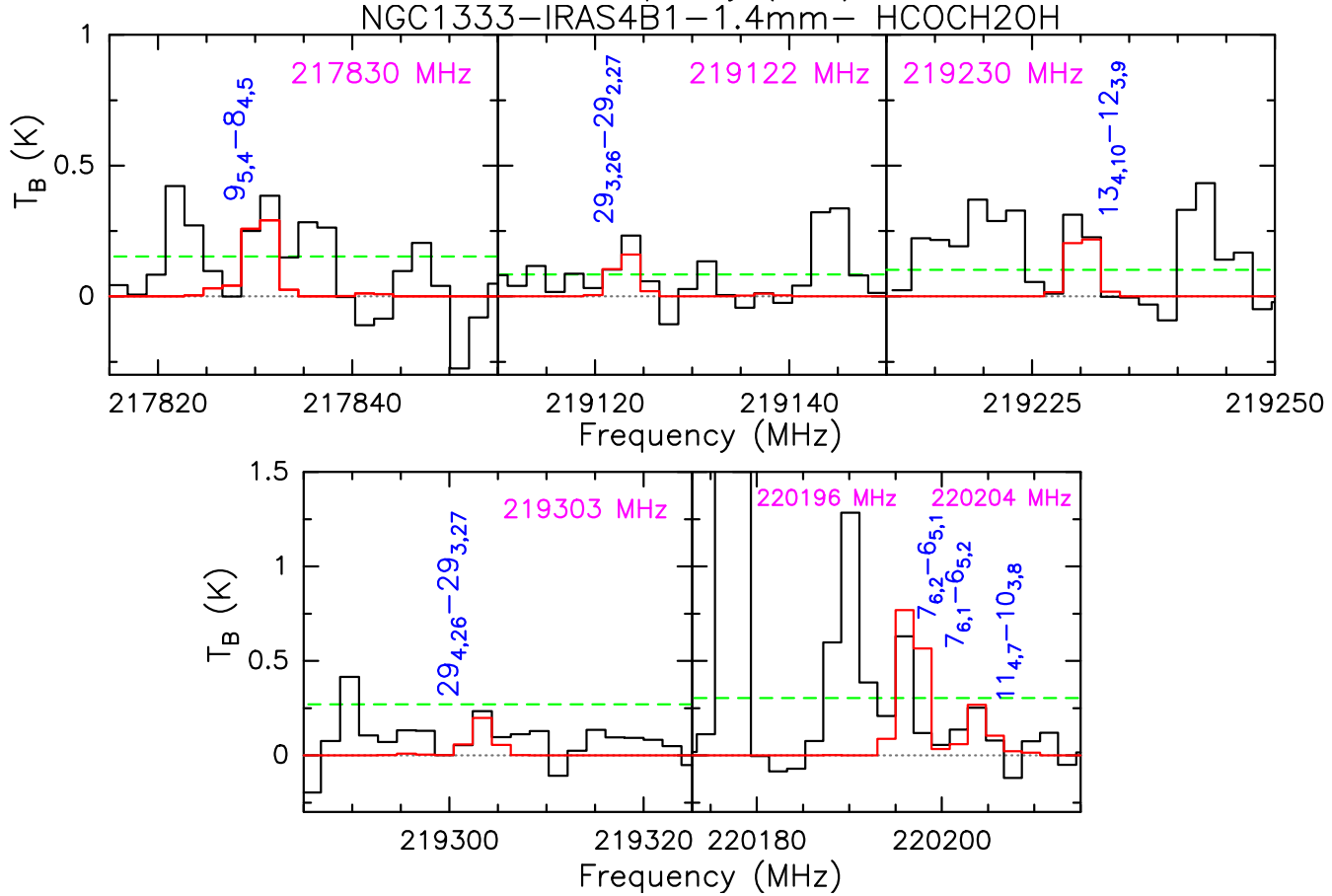

Fig. A.5. Glycolaldehyde emission lines (in $T_{\mathrm{B}}$ scale) detected in the $1.3 \mathrm{~mm}$ and $1.4 \mathrm{~mm}$ spectral windows towards NGC 1333-IRAS4B1 and used to perform the LTE analysis. The horizontal green dotted lines show the $3 \sigma$ level. In blue we mark the glycolaldehyde lines extracted from Table A.3. The red line shows the synthetic spectrum obtained with the GILDAS-Weeds package (Maret et al. 2011) and assuming the rotation diagram solutions (see Fig. 3). 

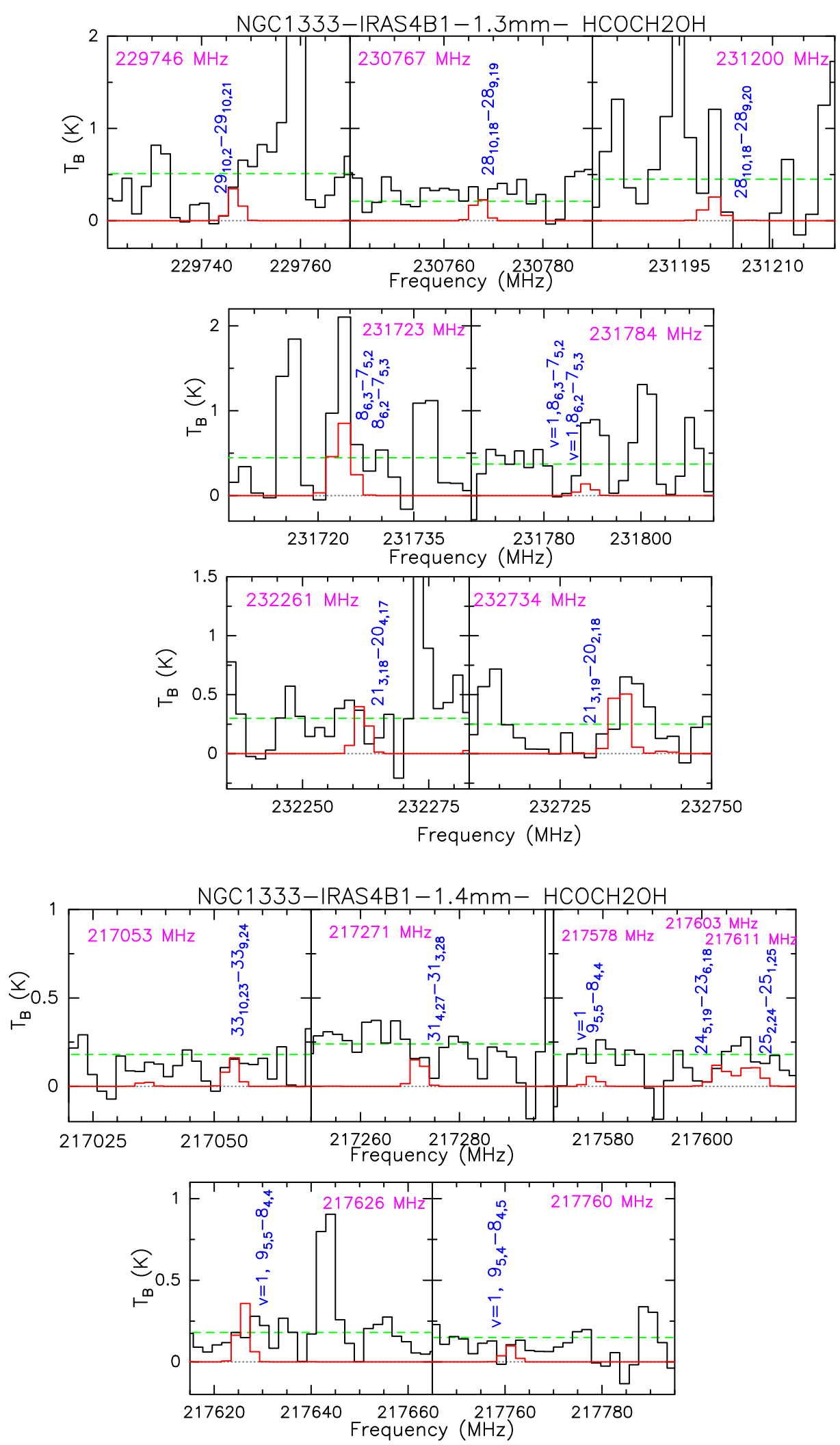

Fig. A.6. Glycolaldehyde emission lines (in $T_{\mathrm{B}}$ scale) observed in the $1.3 \mathrm{~mm}$ and $1.4 \mathrm{~mm}$ spectral windows towards NGC 1333-IRAS4B1 and excluded from the LTE analysis because of severe blending with other emission lines. The horizontal green dotted lines show the $3 \sigma$ level. In blue we mark the glycolaldehyde transitions. The red line shows the synthetic spectrum obtained with the GILDAS-Weeds package (Maret et al. 2011) and assuming the rotation diagram solutions (see Fig. 3). 
M. De Simone et al.: Glycolaldehyde in Perseus young solar analogs

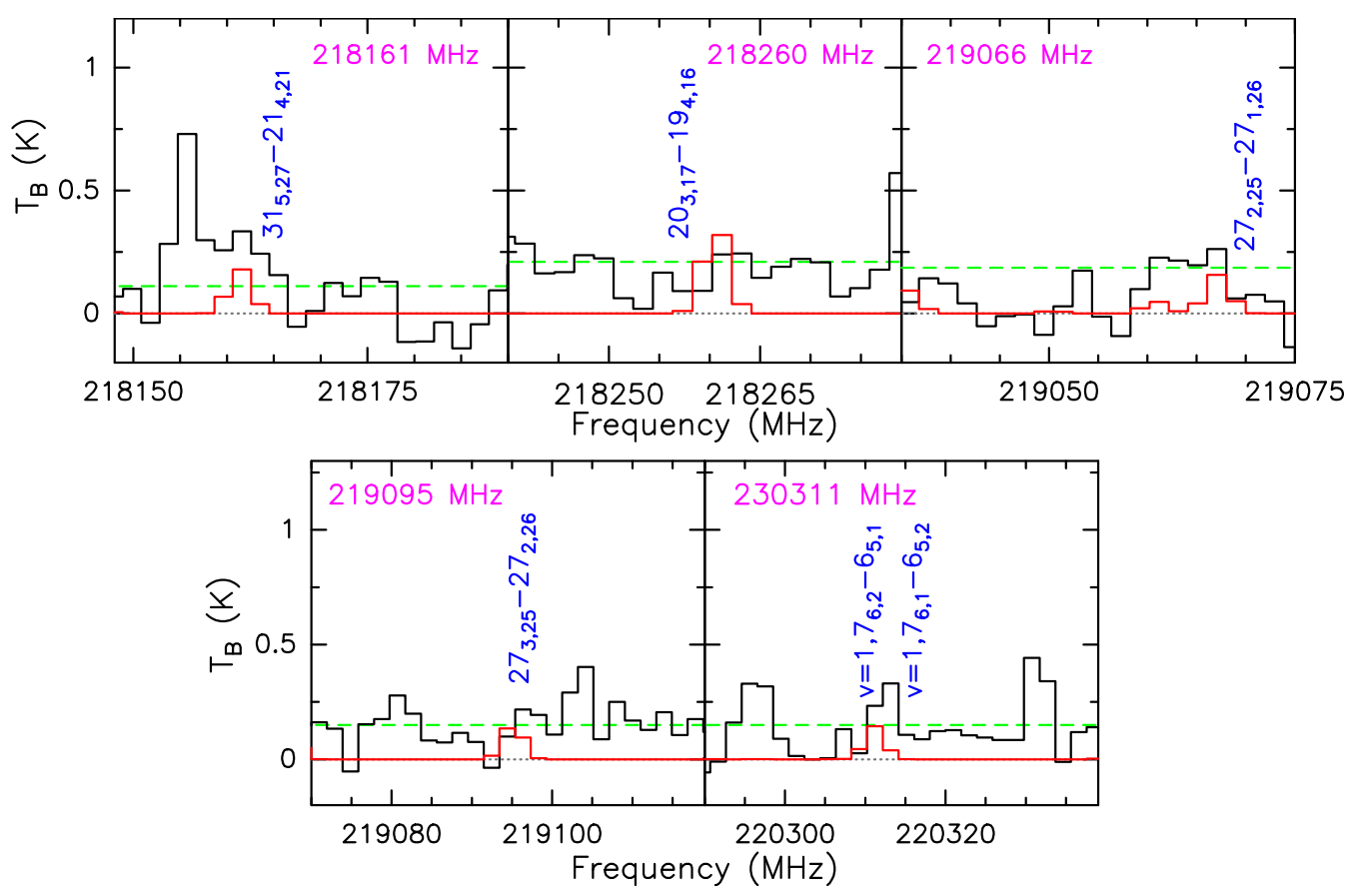

Fig. A.6. continued. 

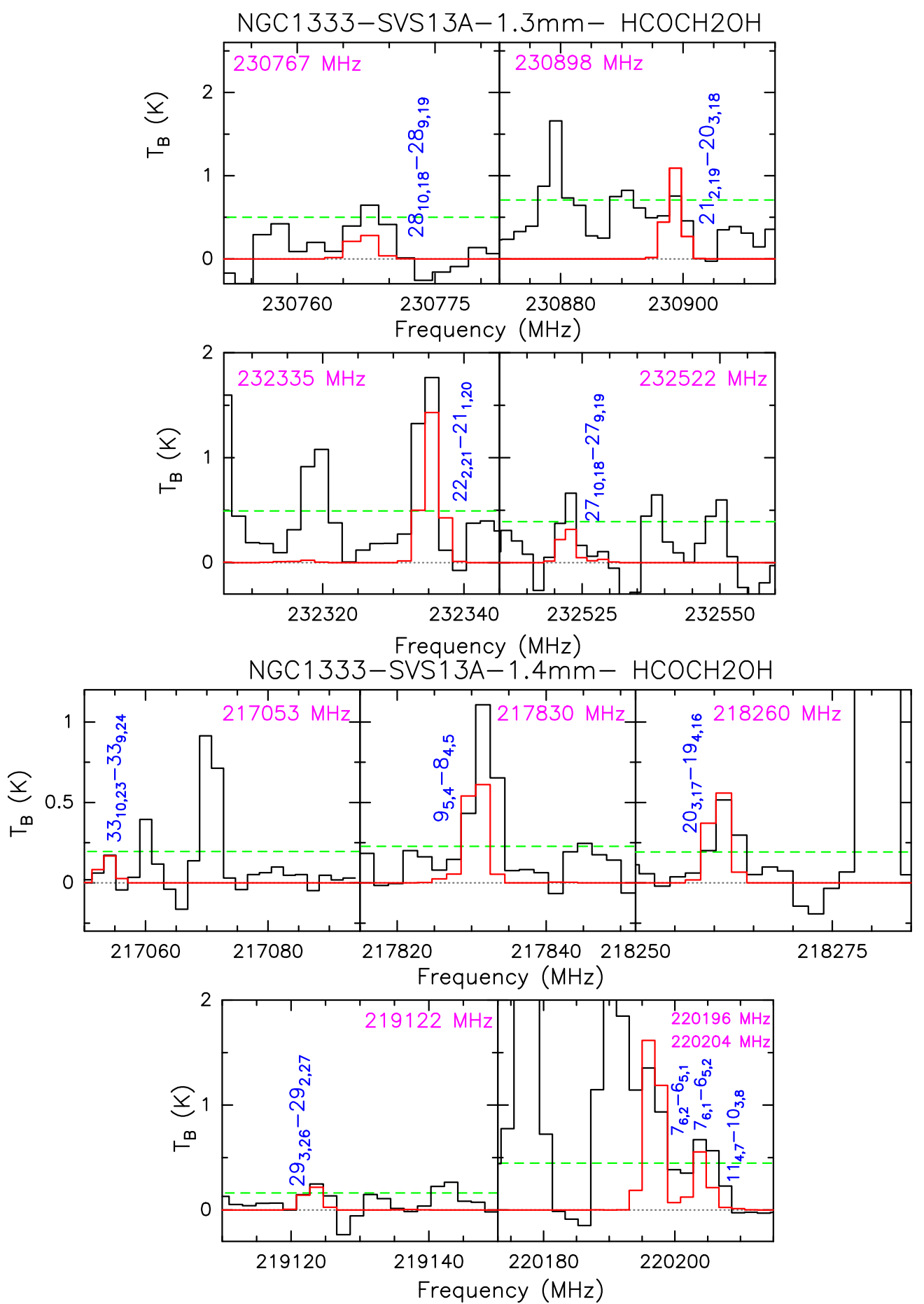

Fig. A.7. Glycolaldehyde emission lines (in $T_{\mathrm{B}}$ scale) detected in the $1.3 \mathrm{~mm}$ and $1.4 \mathrm{~mm}$ spectral windows towards NGC 1333-SVS13A and used to perform the LTE analysis. The horizontal green dotted lines show the $3 \sigma$ level. In blue we mark the glycolaldehyde lines extracted from Table A.4. The red line shows the synthetic spectrum obtained with the GILDAS-Weeds package (Maret et al. 2011) and assuming the rotation diagram solutions (see Fig. 3). 
M. De Simone et al.: Glycolaldehyde in Perseus young solar analogs
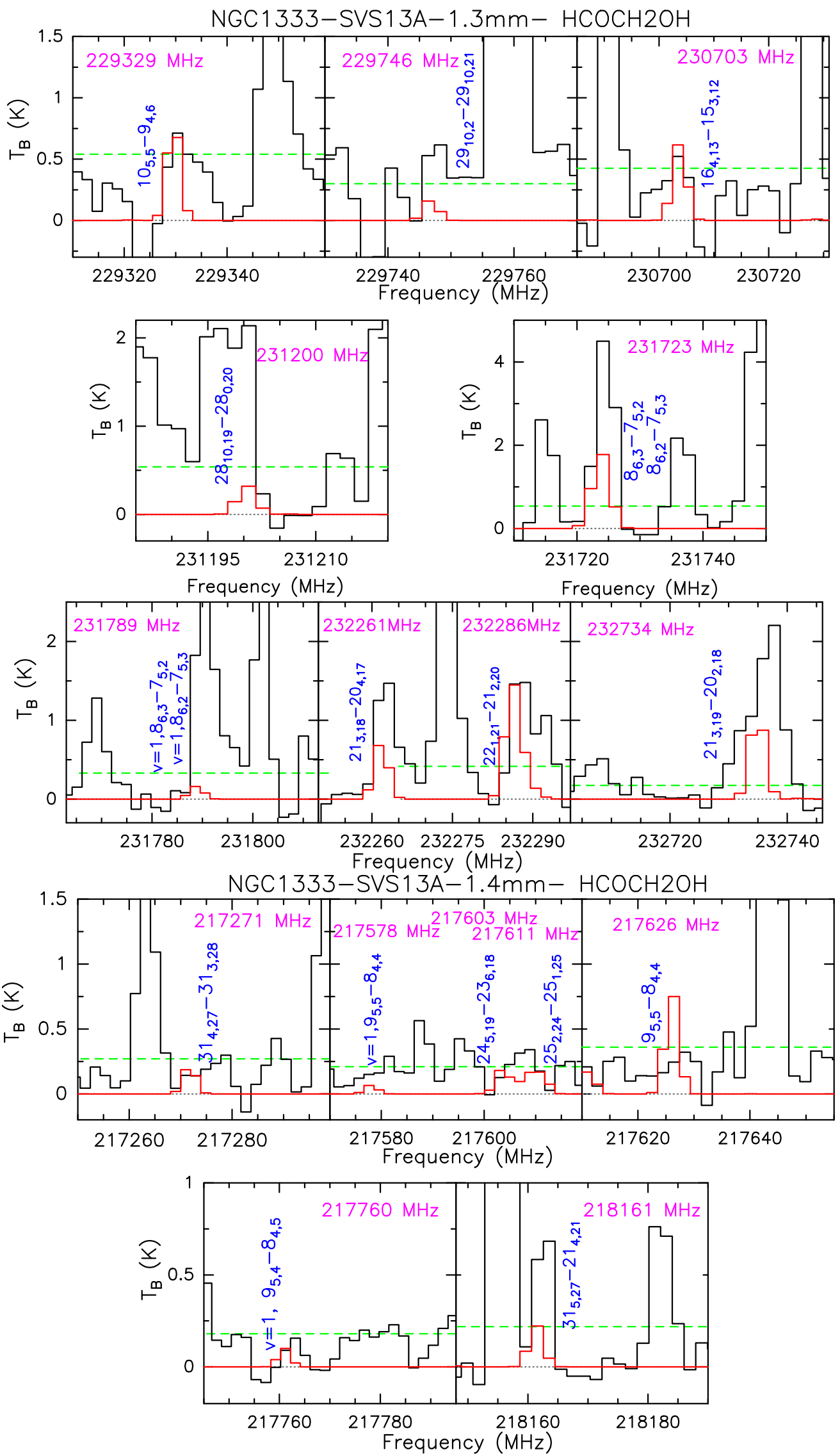

Fig. A.8. Glycolaldehyde emission lines (in $T_{\mathrm{B}}$ scale) observed in the $1.3 \mathrm{~mm}$ and $1.4 \mathrm{~mm}$ spectral windows towards NGC 1333-SVS13A and excluded from the LTE analysis because of severe blending with other emission lines. The horizontal green dotted lines show the $3 \sigma$ level. In blue we mark the glycolaldehyde transitions. The red line shows the synthetic spectrum obtained with the GILDAS-Weeds package (Maret et al. 2011) and assuming the rotation diagram solutions (see Fig. 3). 

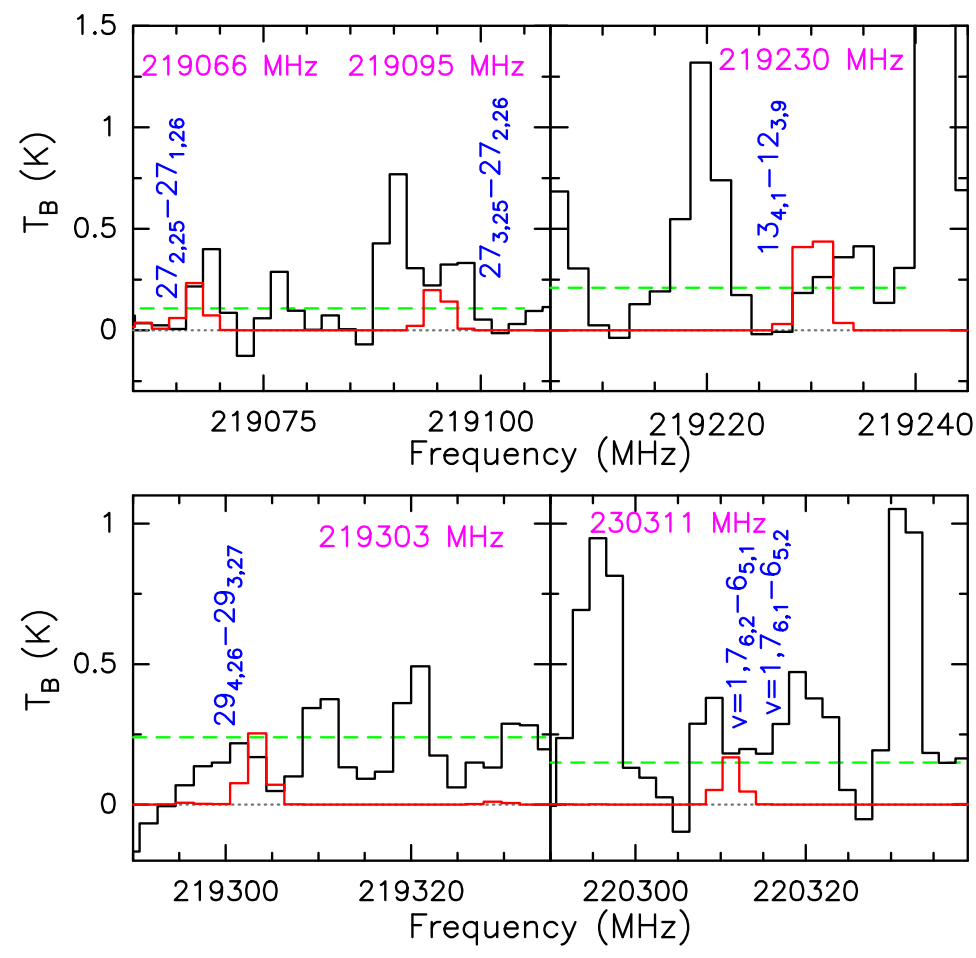

Fig. A.8. continued.
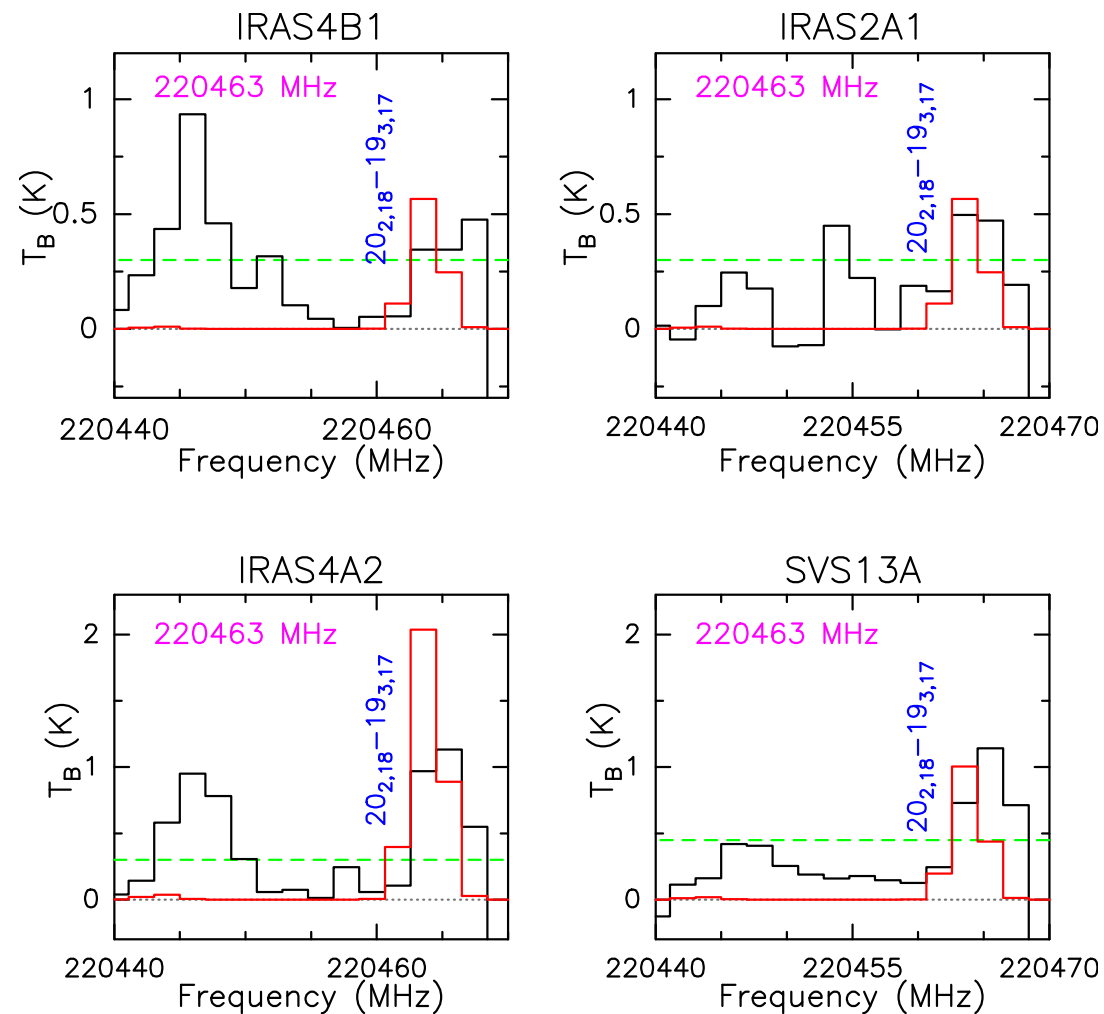

Fig. A.9. Glycolaldehyde $20_{2,18}-19_{3,17}$ emission lines (in $T_{\mathrm{B}}$ scale) observed at $220463 \mathrm{MHz}$ towards NGC 1333-IRAS4B1, -IRAS2A1, IRAS4A2, and -SVS13A, and conservatively excluded from the LTE analysis falling at the edge of the observed WideX band. The horizontal green dotted lines show the $3 \sigma$ level. In blue we mark the glycolaldehyde transitions. The red line shows the synthetic spectrum obtained with the GILDAS-Weeds package (Maret et al. 2011) and assuming the rotation diagram solutions (see Fig. 3). 
M. De Simone et al.: Glycolaldehyde in Perseus young solar analogs

Table A.1. Glycolaldehyde emission lines detected towards NGC 1333 IRAS2A1 (in $T_{\mathrm{B}}$ scale).

\begin{tabular}{lcccccccc}
\hline \hline Transition & $\begin{array}{c}v^{a} \\
(\mathrm{MHz})\end{array}$ & $\begin{array}{c}E_{\mathrm{u}}{ }^{a} \\
(\mathrm{~K})\end{array}$ & $\begin{array}{c}S \mu^{2 a} \\
\left(D^{2}\right)\end{array}$ & $\begin{array}{c}\int T \mathrm{~d}^{b} \\
\left(\mathrm{~K} \mathrm{~km} \mathrm{~s}^{-1}\right)\end{array}$ & $\begin{array}{c}V_{\text {peak }}{ }^{b} \\
\left(\mathrm{~km} \mathrm{~s}^{-1}\right)\end{array}$ & $\begin{array}{c}F W H M^{b} \\
\left(\mathrm{~km} \mathrm{~s}^{-1}\right)\end{array}$ & $\begin{array}{c}T_{\text {peak }}{ }^{b} \\
(\mathrm{mK})\end{array}$ & $\begin{array}{c}\mathrm{rms} \\
(\mathrm{mK})\end{array}$ \\
\hline $31_{4,27}-31_{3,28}$ & 217271.520 & 290.40 & 63.50 & $1.8(1.0)$ & $+6.5(1.2)$ & $4.1(2.9)$ & $434(32)$ & 125 \\
$27_{3,25}-27_{2,26}$ & 219095.117 & 207.92 & 31.71 & $2.3(0.6)$ & $+7.4(0.6)$ & $4.9(1.6)$ & $438(32)$ & 104 \\
$7_{6,2}-6_{5,1}$ & 220196.605 & 37.41 & 29.67 & $10.5(3.8)$ & $+7.8(0.9)$ & $6.5(2.8)$ & $1499(32)$ & 100 \\
$7_{6,1}-6_{5,2}$ & 220196.800 & 37.41 & 29.67 & & & & & \\
$11_{4,7}-10_{3,8}$ & 220204.039 & 46.61 & 18.68 & $4.3(2.8)$ & $+8.0(1.9)$ & $7.0(6.4)$ & $578(31)$ & 100 \\
$7_{6,2}-6_{5,1} v=1$ & 220311.082 & 318.20 & 29.90 & $2.7(1.4)$ & $+6.9(1.6)$ & $5.8(3.2)$ & $422(34)$ & 138 \\
$7_{6,1}-6_{5,2} v=1$ & 220311.251 & 318.20 & 29.90 & & & & & \\
\hline & & & & $1.3 \mathrm{~mm}$ & & & & \\
\hline $21_{2,19}-20_{3,18}$ & 230898.536 & 131.19 & 71.15 & $4.2(2.2)$ & $+6.0(1.6)$ & $5.9(3.4)$ & $670(80)$ & 167 \\
$8_{6,3}-7_{5,2}$ & 231723.266 & 41.85 & 29.86 & $16.6(4.7)$ & $+7.2(1.2)$ & $8.3(3.4)$ & $1954(104)$ & 220 \\
$8_{6,2}-7_{5,3}$ & 231724.332 & 41.85 & 29.86 & & & & & \\
$22_{2,21}-21_{1,20}$ & 232335.395 & 134.52 & 94.55 & $8.9(2.5)$ & $+7.8(0.6)$ & $4.6(1.6)$ & $1820(254)$ & 232 \\
$27_{10,18}-27_{9,19}$ & 232522.255 & 271.37 & 77.57 & $2.9(1.9)$ & $+8.4(2.0)$ & $6.1(4.8)$ & $454(78)$ & 107 \\
$21_{3,19}-20_{2,18}$ & 232734.940 & 131.22 & 71.21 & $6.9(1.6)$ & $+6.2(0.6)$ & $4.8(1.1)$ & $1348(93)$ & 10 \\
\hline
\end{tabular}

Notes. ${ }^{(a)}$ From the Jet Propulsion Laboratory database (Pickett et al. 1998). ${ }^{(b)}$ The errors are the Gaussian fit uncertainties. For the weakest lines, the systemic velocity (see Table 1) and typical linewidths are assumed and consequently no error on these parameters is quoted.

Table A.2. Glycolaldehyde emission lines detected towards NGC 1333 SVS13-A (in $T_{\mathrm{B}}$ scale).

\begin{tabular}{lcccccccc}
\hline \hline Transition $^{a}$ & $\begin{array}{c}v^{a} \\
(\mathrm{MHz})\end{array}$ & $\begin{array}{c}E_{\mathrm{u}}{ }^{a} \\
(\mathrm{~K})\end{array}$ & $\begin{array}{c}S \mu^{2 a} \\
\left(D^{2}\right)\end{array}$ & $\begin{array}{c}\int T \mathrm{~d}^{b} \\
\left(\mathrm{~K} \mathrm{~km} \mathrm{~s}^{-1}\right)\end{array}$ & $\begin{array}{c}V_{\text {peak }}{ }^{b} \\
\left(\mathrm{~km} \mathrm{~s}^{-1}\right)\end{array}$ & $\begin{array}{c}F W H M^{b} \\
\left(\mathrm{~km} \mathrm{~s}^{-1}\right)\end{array}$ & $\begin{array}{c}T_{\text {peak }}{ }^{b} \\
(\mathrm{mK})\end{array}$ & $\begin{array}{c}\mathrm{rms} \\
(\mathrm{mK})\end{array}$ \\
\hline $33_{10,23}-33_{9,24}$ & 217053.668 & 374.45 & 104.58 & $0.6(0.2)$ & $+8.4(0.0)$ & $3.5(0.0)$ & $184(21)$ & 65 \\
$9_{5,4}-8_{4,5}$ & 217830.692 & 40.20 & 25.16 & $5.2(0.5)$ & $+7.0(0.7)$ & $4.0(1.4)$ & $1096(36)$ & 76 \\
$20_{3,17}-19_{4,16}$ & 218260.540 & 126.14 & 43.31 & $2.3(0.7)$ & $+7.1(0.7)$ & $4.5(1.4)$ & $455(70)$ & 64 \\
$29_{3,26}-29_{2,27}$ & 219122.861 & 247.80 & 47.54 & $1.3(0.5)$ & $+7.4(1.0)$ & $4.7(1.5)$ & $270(18)$ & 54 \\
$7_{6,2}-6_{5,1}$ & 220196.605 & 37.41 & 29.67 & $5.6(1.8)$ & $+8.5(0.0)$ & $3.5(0.0)$ & $1518(268)$ & 149 \\
$7_{6,1}-6_{5,2}$ & 220196.800 & 37.41 & 29.67 & & & & & \\
$11_{4,7}-10_{3,8}$ & 220204.039 & 46.61 & 18.68 & $2.5(1.7)$ & $+8.4(0.0)$ & $3.5(0.0)$ & $685(268)$ & 149 \\
\hline & & & & $1.3 \mathrm{~mm}$ & & & \\
\hline $28_{10,18}-28_{9,19}$ & 230767.152 & 287.09 & 81.84 & $3.9(1.2)$ & $+7.3(0.9)$ & $5.7(1.9)$ & $630(221)$ & 167 \\
$21_{2,19}-20_{3,18}$ & 230898.536 & 131.19 & 71.15 & $3.9(1.5)$ & $+8.4(0.0)$ & $4.0(0.0)$ & $864(164)$ & 236 \\
$22_{2,21}-21_{1,20}$ & 232335.395 & 134.52 & 94.55 & $8.5(1.8)$ & $+9.3(0.3)$ & $3.8(1.5)$ & $2097(45)$ & 164 \\
$27_{10,18}-27_{9,19}$ & 232522.255 & 271.37 & 77.57 & $3.0(0.9)$ & $+8.0(0.7)$ & $4.3(1.5)$ & $697(54)$ & 130 \\
\hline
\end{tabular}

Notes. ${ }^{(a)}$ From the Jet Propulsion Laboratory database (Pickett et al. 1998). ${ }^{(b)}$ The errors are the Gaussian fit uncertainties. For the weakest lines, the systemic velocity (see Table 1) and typical linewidths are assumed and consequently no error on these parameters is quoted. 
Table A.3. Glycolaldehyde emission lines detected towards NGC 1333 IRAS4A2 (in $T_{\mathrm{B}}$ scale).

\begin{tabular}{lcccccccc}
\hline \hline Transition $^{a}$ & $\begin{array}{c}v^{a} \\
(\mathrm{MHz})\end{array}$ & $\begin{array}{c}E_{\mathrm{u}}{ }^{a} \\
(\mathrm{~K})\end{array}$ & $\begin{array}{c}S \mu^{2 a} \\
\left(D^{2}\right)\end{array}$ & $\begin{array}{c}\int T \mathrm{~d}^{b} \\
\left(\mathrm{~K} \mathrm{~km} \mathrm{~s}^{-1}\right)\end{array}$ & $\begin{array}{c}V_{\text {peak }}{ }^{b} \\
\left(\mathrm{~km} \mathrm{~s}^{-1}\right)\end{array}$ & $\begin{array}{c}F W H M^{b} \\
\left(\mathrm{~km} \mathrm{~s}^{-1}\right)\end{array}$ & $\begin{array}{c}T_{\text {peak }}{ }^{b} \\
(\mathrm{mK})\end{array}$ & $\begin{array}{c}\mathrm{rms} \\
(\mathrm{mK})\end{array}$ \\
\hline $33_{10,23}-33_{9,24}$ & 217053.668 & 374.45 & 104.58 & $2.6(1.6)$ & $+7.0(0.0)$ & $3.5(0.0)$ & $701(61)$ & 80 \\
$39_{10,30}-38_{11,27}$ & 217626.061 & 40.20 & 25.19 & $4.2(1.1)$ & $+5.9(0.6)$ & $4.5(1.3)$ & $876(20)$ & 90 \\
$9_{5,4}-8_{4,5}$ & 217830.692 & 40.20 & 25.16 & $5.9(1.6)$ & $+5.7(1.0)$ & $7.7(2.8)$ & $699(67)$ & 70 \\
$20_{3,17}-19_{4,16}$ & 218260.540 & 126.14 & 43.31 & $4.7(0.8)$ & $+4.7(0.5)$ & $5.7(1.2)$ & $784(58)$ & 69 \\
$27_{2,25}-27_{1,26}$ & 219066.914 & 207.91 & 31.72 & $4.3(1.8)$ & $+5.4(0.9)$ & $5.2(3.0)$ & $778(78)$ & 60 \\
$29_{3,26}-29_{2,27}$ & 219122.861 & 247.80 & 47.54 & $2.4(1.2)$ & $+6.2(1.2)$ & $5.4(3.0)$ & $404(63)$ & 49 \\
$29_{4,26}-29_{3,27}$ & 219303.367 & 247.81 & 47.54 & $2.8(0.8)$ & $+7.0(0.0)$ & $3.5(0.0)$ & $777(23)$ & 26 \\
$7_{6,2}-6_{5,1}$ & 220196.605 & 37.41 & 29.67 & $7.4(1.9)$ & $+7.0(0.0)$ & $3.5(0.0)$ & $1969(256)$ & 49 \\
$7_{6,1}-6_{5,2}$ & 220196.800 & 37.41 & 29.67 & & & & \\
$11_{4,7}-10_{3,8}$ & 220204.039 & 46.61 & 18.68 & $3.2(1.9)$ & $+7.0(0.0)$ & $3.5(0.0)$ & $885(256)$ & 49 \\
\hline & & & & $1.3 \mathrm{~mm}$ & & & & \\
\hline $10_{5,5}-9_{4,6}$ & 229329.580 & 45.76 & 25.44 & $3.6(1.2)$ & $+7.0(0.0)$ & $3.5(0.0)$ & $957(199)$ & 120 \\
$16_{4,13}-15_{3,12}$ & 230703.712 & 85.68 & 27.38 & $4.6(2.9)$ & $+6.7(1.5)$ & $3.8(2.2)$ & $1102(197)$ & 180 \\
$28_{10,18}-28_{9,19}$ & 230767.152 & 287.09 & 81.84 & $3.1(1.2)$ & $+7.0(0.0)$ & $3.5(0.0)$ & $866(149)$ & 200 \\
$21_{2,19}-20_{3,18}$ & 230898.536 & 131.19 & 71.15 & $6.9(2.3)$ & $+7.0(0.0)$ & $3.5(0.0)$ & $1887(133)$ & 181 \\
$21_{3,18}-20_{4,17}$ & 232261.725 & 137.84 & 49.86 & $8.6(3.6)$ & $+6.1(0.9)$ & $3.9(2.0)$ & $2021(310)$ & 160 \\
$22_{1,21}-21_{2,20}$ & 232286.032 & 134.52 & 94.55 & $12.8(3.7)$ & $+5.9(0.6)$ & $3.9(1.3)$ & $3031(31)$ & 150 \\
$22_{2,21}-21_{1,20}$ & 232335.395 & 134.52 & 94.55 & $21.9(2.6)$ & $+7.2(0.3)$ & $3.7(0.7)$ & $5592(23)$ & 211 \\
$27_{10,18}-27_{9,19}$ & 232522.255 & 271.37 & 77.57 & $9.4(2.2)$ & $+7.4(0.5)$ & $4.7(1.3)$ & $1861(62)$ & 124 \\
\hline
\end{tabular}

Notes. ${ }^{(a)}$ From the Jet Propulsion Laboratory database (Pickett et al. 1998). ${ }^{(b)}$ The errors are the Gaussian fit uncertainties. For the weakest lines, the systemic velocity (see Table 1) and typical linewidths are assumed and consequently no error on these parameters is quoted.

Table A.4. Glycolaldehyde emission lines detected towards NGC 1333 IRAS4B1 (in $T_{\mathrm{B}}$ scale).

\begin{tabular}{|c|c|c|c|c|c|c|c|c|}
\hline Transition $^{a}$ & $\begin{array}{c}v^{a} \\
(\mathrm{MHz}) \\
\end{array}$ & $\begin{array}{l}E_{\mathrm{u}}{ }^{a} \\
(\mathrm{~K})\end{array}$ & $\begin{array}{l}S \mu^{2 a} \\
\left(D^{2}\right)\end{array}$ & $\begin{array}{c}\int T \mathrm{~d} V^{b} \\
\left(\mathrm{~K} \mathrm{~km} \mathrm{~s}^{-1}\right)\end{array}$ & $\begin{array}{c}V_{\text {peak }}^{b} \\
\left(\mathrm{~km} \mathrm{~s}^{-1}\right)\end{array}$ & $\begin{array}{r}F W H M^{b} \\
\left(\mathrm{~km} \mathrm{~s}^{-1}\right)\end{array}$ & $\begin{array}{l}T_{\text {peak }}^{b} \\
(\mathrm{mK})\end{array}$ & $\begin{array}{c}\mathrm{rms} \\
(\mathrm{mK})\end{array}$ \\
\hline \multicolumn{9}{|c|}{$1.4 \mathrm{~mm}$} \\
\hline $9_{5,4}-8_{4,5}$ & 217830.692 & 40.20 & 25.16 & $1.8(0.5)$ & $+7.0(0.0)$ & $3.5(0.0)$ & $485(23)$ & 51 \\
\hline $29_{3,26}-29_{2,27}$ & 219122.861 & 247.80 & 47.54 & $1.0(0.5)$ & $+6.2(1.2)$ & $4.2(1.9)$ & $226(7)$ & 28 \\
\hline $13_{4,10}-12_{3,9}$ & 219230.248 & 60.52 & 21.81 & $1.6(0.6)$ & $+7.4(0.8)$ & $3.5(0.0)$ & $366(17)$ & 34 \\
\hline $29_{4,26}-29_{3,27}$ & 219303.367 & 247.81 & 47.54 & $0.9(0.4)$ & $+7.0(0.0)$ & $3.5(0.0)$ & $253(23)$ & 90 \\
\hline $7_{6,2}-6_{5,1}$ & 220196.605 & 37.41 & 29.67 & $2.5(0.5)$ & +83 & 3.70 & 101) & 101 \\
\hline $7_{6,1}-6_{5,2}$ & 220196.800 & 37.41 & 29.67 & $2 . J(0 . J)$ & $+0.3(0 . J)$ & $5.1(0.0)$ & $04 x(101)$ & 101 \\
\hline $11_{4,7}-10_{3,8}$ & 220204.039 & 46.61 & 18.68 & $1.3(0.6)$ & $+7.8(1.0)$ & $4.6(2.2)$ & 251(101) & 101 \\
\hline \multicolumn{9}{|c|}{$1.3 \mathrm{~mm}$} \\
\hline $10_{5,5}-9_{4,6}$ & 229329.580 & 45.76 & 25.44 & $3.7(0.9)$ & $+6.2(0.8)$ & $5.2(1.6)$ & $638(65)$ & 102 \\
\hline $16_{4,13}-15_{3,12}$ & 230703.712 & 85.68 & 27.38 & $3.4(1.8)$ & $+6.1(1.4)$ & $5.2(2.8)$ & $631(56)$ & 206 \\
\hline $21_{2,19}-20_{3,18}$ & 230898.536 & 131.19 & 71.15 & $2.2(0.9)$ & $+6.2(0.9)$ & $3.7(1.5)$ & $551(25)$ & 127 \\
\hline $22_{1,21}-21_{2,20}$ & 232286.032 & 134.52 & 94.55 & $2.9(0.9)$ & $+7.0(0.0)$ & $3.5(0.0)$ & $699(124)$ & 124 \\
\hline $22_{2,21}-21_{1,20}$ & 232335.395 & 134.52 & 94.55 & $4.0(0.9)$ & $+7.0(0.0)$ & $3.5(0.0)$ & $932(164)$ & 148 \\
\hline $27_{10,18}-27_{9,19}$ & 232522.255 & 271.37 & 77.57 & $1.8(0.9)$ & $+8.3(1.4)$ & $4.5(2.8)$ & $387(108)$ & 108 \\
\hline
\end{tabular}

Notes. ${ }^{(a)}$ From the Jet Propulsion Laboratory database (Pickett et al. 1998). ${ }^{(b)}$ The errors are the Gaussian fit uncertainties. For the weakest lines, the systemic velocity (see Table 1) and typical linewidths are assumed and consequently no error on these parameters is quoted. 